\title{
Individual versus Group Choices of Repeated Game Strategies: A Strategy Method Approach
}

\author{
Timothy N. Cason ${ }^{1}$ and Vai-Lam Mui ${ }^{2}$
}

\begin{abstract}
:
We study experimentally the indefinitely repeated noisy prisoner's dilemma, in which random events can change an intended action to its opposite. We investigate whether groups choose Always Defect less and use lenient or forgiving strategies more than individuals, and how decision-makers experiment with different strategies by letting them choose from an extensive list of repeated game strategies. We find that groups use forgiving and tit-for-tat strategies more than individuals. Always Defect, however, is the most popular strategy for both groups and individuals. Groups and individuals cooperate at similar rates overall, and they seldom experiment with different strategies in later supergames.
\end{abstract}

Keywords: Laboratory Experiment; Cooperation; Repeated Games; Strategy

JEL Codes: C73; C92

Acknowledgement: We are grateful to the Krannert School of Management of Purdue University for financial support. For valuable comments and suggestions we thank two anonymous referees and an advisory editor, Klaus Abbink, Nick Feltovich, Phil Grossman, Andreas Leibbrandt, Matt Leister, Birendra Rai, Anmol Ratan, Yaroslav Rosokha, Brock Stoddard, Erte Xiao, seminar audiences at Cologne, Düsseldorf, Florida State, Goethe, Monash, Pittsburgh, Purdue, and Southern Methodist Universities, and conference participants at the Australasian Public Choice Conference, the Canadian Economic Association, the Economic Science Association, Xiamen University, and the Monash Workshop on "Macroeconomics, Experimental Economics, and Behavior." Huanren Zhang provided valuable research assistance. We alone are responsible for any errors.

${ }^{1}$ Department of Economics, Purdue University, 403 W. State St., West Lafayette, IN 479072056, U.S.A.

${ }^{2}$ Department of Economics, Monash Business School, Monash University, P.O. Box 11E, Clayton, Victoria 3800, Australia

(C) 2019 Timothy N. Cason and Vai-Lam Mui

All rights reserved. No part of this paper may be reproduced in any form, or stored in a retrieval system, without the prior written permission of the author.

monash.edu/ businesseconomics

ABN 12377614012 CRICOS Provider No. 00008C

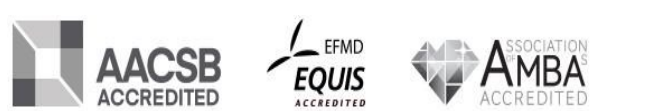




\section{Introduction}

Repeated interactions are pervasive in economics, ranging from the interactions between employers and employees, trading partners, to nation states. Furthermore, noise is often present in such interactions. For example, in a repeated joint project, random shocks may negatively affect the quality of the work delivered by a person to her partner despite her high effort, but her partner can only observe the quality of the work delivered but not the effort devoted. In recent reflection of his classic tournament study, Axelrod (2012, p. 22) emphasizes that "some degree of noise is typical of most strategic interactions," and observes that an important omission in Axelrod (1984) is that it does not allow for the possibility "that a choice by one player would occasionally be misreported to the other" (Axelrod, 2012, p. 22). This paper presents an experiment to study decision-makers' strategy choices in an infinitely repeated noisy prisoner's dilemma, in which a decision-maker's chosen action can be switched randomly to the other action, but the opponent only observes the realized action.

Our experiment has two important features. First, similar to several recent studies on indefinitely repeated games (Embrey et al., 2016; Dal Bó and Fréchette, 2018b; Romero and Rosokha, 2018), it uses a Strategy Method design that allows decision-makers to explicitly choose their repeated game strategies (Axelrod, 1984; Selten et al., 1997). This allows us to gather novel evidence to compare how often players experiment with different strategies in the repeated noisy prisoner's dilemma. Second, we study decision-makers who are individuals and groups. This allows us to investigate whether groups behave more sophisticatedly and more often choose forgiving and lenient strategies and avoid low-performing strategies such as Always Defect.

We design our experiment to study the following research questions:

Question 1. Do groups use different strategies than individuals? How often do decisionmakers choose "slow to anger" or "fast to forgive" strategies in this strategy method environment?

Question 2. How often do decision-makers experiment with new strategies in this strategy method environment? Are groups more likely to experiment than individuals?

Question 3. At what rates do decision-makers cooperate in this strategy method environment? Are groups more likely to cooperate than individuals?

Question 4. Is the Always Defect strategy still the most popular strategy in this strategy method environment? Are groups less likely to choose Always Defect than individuals?

These questions are motivated by several considerations. In a recent experimental study on 
the infinitely repeated noisy prisoner's dilemma, Fudenberg et al. (2012) report that decisionmakers often adopt lenient strategies that do not immediately retaliate after the first defection or forgiving strategies that return to cooperation after inflicting some punishment. Always Defect, however, despite its poor performance, is nevertheless the most popular strategy chosen. Aoyagi et al. (2018) compares behavior in the repeated noisy prisoner's dilemma with public monitoring (in which information about past actions is noisy but public as in Fudenberg et al. (2012) to private monitoring (in which information about past action is noisy but private). In both treatments, popular strategies include lenient and forgiving strategies, but Always Defect again is a very popular strategy.

Given the complexity of the infinitely repeated noisy prisoner's dilemma, learning is likely to be important in affecting decision-makers' choices of repeated game strategies. Decisionmakers who seldom experiment with different strategies, however, are unlikely to get useful information that facilitates learning. Information about the payoffs of different strategies and the strategies' relative returns is only generated when decision-makers experiment with alternative strategies (Merlo and Schotter, 1992). ${ }^{1}$ A good understanding of how decision-makers experiment with different repeated game strategies is therefore an important step for understanding how learning affects behavior. Both Fudenberg et al. (2012) and Aoyagi et al. (2018) adopt the Direct Response Method in which each subject chooses Cooperate and Defect in each round of a repeated game. They then use the Strategy Frequency Estimation Method introduced by Dal Bó and Fréchette (2011) to estimate the frequency each strategy was chosen. In this method, researchers assume that each decision-maker chooses a fixed strategy across all supergames being analyzed, and then use Maximum Likelihood to estimate the proportion of different strategies being played. Because this approach assumes that each decision-maker chooses a fixed strategy across all supergames under consideration, by design this approach cannot investigate whether and how they experiment with different strategies. Moreover, even if this method were applied to subsets of the data to study how strategy choices change over time, the comparison must be made at the aggregate level. For individual decision-makers the method only returns a likelihood of different strategy classifications so transitions from one strategy choice to another cannot be observed directly.

\footnotetext{
${ }^{1}$ Information about the repeated game strategy choices of others could provide additional understanding about why a particular strategy performs differently when matched with alternative opponent strategies. We did not provide this information, however, since it is rarely observable in practice.
} 
Fudenberg et al. (2012) found that subjects who chose Always Defect earn substantially less than those who chose conditionally cooperative strategies such as Tit-for-Tat and its variants (e.g., 2-Tits-for-2-Tats, which punishes two times after two defections and is both lenient and forgiving). Fudenberg and Levine (2016, p.164) argue that this suggests that decision-makers find it hard to learn which strategies will do well in the repeated noisy prisoner's dilemma, "both because of the large size of their own strategy space and the many possible strategies their opponents might be using." Our study restricts each subject to choose one of the twenty strategies that Fudenberg et al. (2012) consider in their estimation, although decision-makers can change strategy choices across supergames. Our design thus allows us to investigate whether reducing the size of a player's own strategy space and that of his opponent reduces heterogeneity of play and facilitates learning, and possibly reduces the adoption of Always Defect.

In our experiment, decision-makers first play four supergames using the direct response method to become familiar with the strategic environment. They then play ten supergames using the strategy method, in which they choose and commit to one of twenty available repeated game strategies at the beginning of each supergame. This allows us to directly observe whether and how decision-makers switch between strategies across supergames. For example, we can investigate whether a decision-maker who chooses a forgiving strategy is more or less likely to switch to another forgiving strategy if she decides to switch. We also directly observe the frequency that a decision-maker who chooses Always Defect switches to a cooperative strategy to shed more light on the important puzzle of the frequent choice of Always Defect despite its poor performance.

We emphasize, however, that our strategy method approach and the existing approach of combining the direct response method with the Strategy Frequency Estimation Method by Dal Bó and Fréchette (2011) and Fudenberg et al. (2012) and others both impose restrictions on the strategy space, though at different stages. The existing approach puts no ex ante restrictions on the strategies that decision-makers can adopt, but relies on the assumption that each adopts a fixed strategy in all supergames. It also imposes ex post restrictions on the set of strategies from which decision-makers are assumed to have chosen for the estimation. On the other hand, our strategy method approach requires us to impose ex ante restrictions on the set of available strategies. Based on theoretical considerations and their data, Fudenberg et al. (2012) focus on a specific set of 20 strategies and they estimate that subjects choose a subset of these strategies. Since our experiment adopts payoff and other parameters used by Fudenberg et al. (2012), we allow decision-makers to 
choose from this same set of 20 strategies in the beginning of each supergame.

The recent literature that employs the laboratory method to study how decision-makers behave in the infinitely repeated prisoner's dilemma (both noisy and deterministic) focus on the case when all decision-makers are individuals. ${ }^{2}$ Many repeated interactions, however, involve decision-makers who are groups. For example, in employment relationships, decisions by a union and the firm are often made by groups of senior leaders. In interactions between nation states, decisions are made by cabinets. ${ }^{3}$ A sizable experimental literature in economics has compared individual to group behavior and finds that overall groups are cognitively more sophisticated and also more self-regarding than individuals (for surveys see Charness and Sutter, 2012a, and Kugler et al., 2012). Research in psychology has also shown than for intellective tasks, groups perform better than individuals (Laughlin and Ellis, 1986; Laughlin et al. 1991; 2002; 2006; also see the review by Kerr and Tindale (2004) and the references cited there). This literature also emphasizes that the better performance of groups in intellective tasks is due to their high demonstrability, in that an individual who has discovered the correct answer can demonstrate this clearly to others (see, for example, Laughlin and Ellis, 1986, and Laughlin et al., 2006).

In Fudenberg et al. (2012) every decision-maker is an individual, and despite its poor performance they find that Always Defect is the most popular strategy. They also find that their subjects do not use cognitively demanding "high-memory" strategies, and conjecture that "cognitive constraints may lead subjects to use relatively simple strategies" (p. 727). They also observe that the Win-Stay-Lose-Shift strategy that is emphasized by evolutionary biologists (Nowak and Sigmund, 1993) and in evolutionary game theory (Fudenberg and Maskin, 1990)-which is to play $\mathrm{C}$ (Cooperate) if last round's outcome was $(C, C)$ or $(D, D)$, otherwise play D (Defect)--is counter-intuitive and is hardly used by subjects. They also examine whether individuals adopt "exploitive" versions of the main cooperative strategies that defect on the first move and then return to the strategy as normally specified (for example, Defect-Tit-for-2-Tats), but conclude that "subjects did not discover the benefit of these exploitive strategies" (p. 741). ${ }^{4}$

\footnotetext{
${ }^{2}$ The question of whether groups and individuals may behave differently was not discussed in the recent survey on experimental studies of infinitely repeated games by Dal Bó and Fréchette (2018a).

${ }^{3}$ In this first exploration of group versus individual play in the infinitely repeated noisy prisoner's dilemma, we consider the case in which a group's decision is made by majority voting, and abstract from the possibility that the preferences of some members of the group may be more important than others.

${ }^{4}$ Citing findings reported in (the working paper version of) Dreber et al. (2014), Fudenberg et al. (2012) argue that social preferences do not seem to be the main factor in explaining subjects' choices of forgiving and lenient strategies in their study. Reviewing several studies that consider the role of social preferences in experimental indefinitely
} 
Compared to a single individual who makes decisions alone in the repeated noisy prisoner's dilemma, a group of individuals who have the opportunity to engage in deliberation may be more likely to recognize that Always Defect can be out-performed by other strategies. Groups may also be more likely to experiment with strategies other than Always Defect, which can allow them to have the opportunity to observe that Always Defect performs significantly worse than other strategies. Groups may thus be less likely to choose Always Defect than individuals, and be more likely to use complex and memory-demanding strategies. Groups may also be more likely to experiment and adopt counter-intuitive strategies such as Win-Stay-Lose-Shift. Groups may also be better than individuals at recognizing the benefit of exploitative strategies.

To empirically evaluate whether group play differs from individual play in the infinitely repeated prisoner's dilemma, our experiment considers two treatments. In the Individual treatment each decision-maker is an individual, while in the Group treatment each decision-maker is a threeperson group who makes decision based on majority rule. To increase both types of decisionmakers' understanding of the expected payoffs of their chosen strategies, the supergame was "played out" round by round. Subjects saw the action and payoff results of each round and had to click to continue through each round.

We find that in both the individual treatment and the group treatment, Always Defect is the most popular strategy and is chosen about 20 percent of the time. Decision-makers also often chose different versions of the tit-for-tat strategy, with the lenient and forgiving two-tits-for-two-tats being the most common choice for groups. Groups and individuals behave similarly in many dimensions: they are equally likely to choose strategies that involve some cooperation (about 77 percent), or some exploitation (about 18 percent), and they do not employ more complex strategies that are based on greater memory length. Compared to individuals, however, groups employ significantly more forgiving strategies (especially those that are exploitive), more tit-for-tat strategies, and fewer grim strategies.

In the first three supergames when decision-makers choose using the strategy method, both individuals and groups experiment with a new strategy about $45 \%$ of the time. This

\footnotetext{
repeated games, Dal Bó and Fréchette (2018a) report that overall, these studies do not find evidence that social preferences are the main driving force of observed behavior. Dal Bó and Fréchette (2018a, p. 88) conclude (in Result 7) that " $(t)$ here is evidence consistent with the idea that the main motivation behind cooperation is strategic." In this first exploration of group versus individual play and strategic experimentation in the indefinitely repeated noisy prisoner's dilemma, we abstract from the investigation of social preferences to focus on other research questions.
} 
experimentation rate drops rapidly, however, and remains low for the later supergames. Across the final four supergames the experimentation rate is about $12 \%$ for both individuals and groups.

We also find that the average rate of cooperation does not differ significantly between groups and individuals, either in the first rounds of each supergame or across all rounds. This highlights that a simple comparison of overall cooperation rates may miss important underlying differences and heterogeneity in strategy choices. As just noted above, groups employ more forgiving and more tit-for-tat strategies than individuals. This does not translate into a higher cooperation rate in the group treatment, however, because groups tend to choose forgiving tit-fortat strategies that involve longer punishment phases and therefore have a lower average rate of cooperation. We also observe some differences in strategy choices for groups and individuals in a control treatment without noisy action implementation, but nearly all of these strategies are cooperative so the overall cooperation rate for both types of decision-makers is very high.

In spite of the relatively high cooperation rate in this repeated noisy prisoner's dilemma with its relatively strong returns to cooperation, Always Defect is still the most popular strategy. This strategy performs poorly when others employ conditionally cooperative strategies, but like Fudenberg et al. (2012) we find that a minority of individuals and groups persist in choosing it. Our strategy method design also enables us to directly observe that these decision-makers seldom try other strategies. Six of the 32 groups are responsible for nearly all of the Always Defect strategy choices, and 7 of the 48 individuals are unwavering Always Defect players. These 13 decisionmakers who choose Always Defect in a majority of the supergames scored significantly worse on the Cognitive Reflection Test compared to others.

Our work complements some recent studies on indefinitely repeated games that allow decision-makers to construct repeated game strategies. Dal Bó and Fréchette (2018b) allow players to construct memory-1 strategies in the repeated (deterministic) prisoner's dilemma by allowing them to condition each period's choice of cooperate or defect on each of the four possible action profiles chosen by decision-makers in the previous period. Also in the repeated prisoner's dilemma, Romero and Rosokha (2018) allow subjects to develop a set of rules that automatically make choices for them. Similar to earlier studies using the direct response method and the Strategy Frequency Estimation Method (e.g., Dal Bó and Fréchette, 2011; Camera and Casari, 2012; Fréchette and Yuksel, 2017), both studies find that the most commonly used strategies are Always Defect, Tit-for-Tat, and Grim. Both studies consider the deterministic prisoner's dilemma instead 
of the noisy prisoner's dilemma that we study, and neither emphasizes decision-makers' experimentation with different repeated game strategies.

Embrey et al. (2016) also allow subjects to construct repeated game strategies, but in indefinitely repeated $4 \times 4$ games that are simplified versions of price- and quantity-setting oligopoly games. Although their experiment allows only a restrictive set of strategies (memory-1, and only conditioning on opponent's previous action), it also allows one-shot deviations so that the strategy space is effectively unrestricted. They find that only 5 of the possible 1024 strategies are chosen more than $5 \%$ of the time. They also find that subjects seldom change their choices of strategies. Interestingly, in our different context of the indefinitely repeated noisy prisoner's dilemma, we find that $72 \%$ of the groups and $79 \%$ of the individuals never change strategies during the last four supergames.

While the extensive literature on group versus individual behavior has studied a wide variety of one-shot games, considerably fewer studies have examined how individuals and groups may behave differently in repeated games, and the existing small number of studies focus on finitely repeated deterministic games. ${ }^{5}$ When the stage game has a unique Nash equilibrium — such as in the prisoner's dilemma and public goods games - finite repetition does not enlarge the set of equilibria. The literature on (individual) finitely repeated prisoner's dilemma and public goods games therefore focuses on determining empirically whether backward induction can lead to the unravelling of cooperation (see, for example, the recent contributions by Cox and Stoddard (2018) on the finitely repeated public goods game and Embrey et al. (2018) on the finitely repeated prisoner's dilemma and the references discussed therein). Reflecting this same focus, the recent contributions that compare individual to group play in the finitely repeated prisoner's dilemma (Kagel and McGee, 2016) and the finitely repeated public goods game (Cox and Stoddard, 2018) also emphasize whether backwards induction is more likely to lead to the unravelling of cooperation in groups than in individuals. Infinite repetition of the deterministic prisoner's dilemma, however, enlarges the set of equilibria and with a sufficiently high discount factor, cooperation can be supported as equilibrium with many different strategies. In the presence of

\footnotetext{
${ }^{5}$ For studies that compare individual to group play in finitely repeated deterministic games, see, for example, Bornstein et al. (2008), Abbink et al. (2010), Ahn et al. (2011), Kroll et al. (2013), Müller and Tan (2013), Cason and Mui (2015), Kagel and McGee (2016), Auerswald et al. (2018), Cox and Stoddard (2018) and Kamei (forthcoming). Gong et al. (2009) compare individual to group play in the finitely repeated prisoner's dilemma, with a different type of uncertainty than we implement.
} 
multiple equilibria, the recent experimental literature on the indefinitely repeated deterministic prisoner's dilemma and the indefinitely repeated noisy prisoner's dilemma discussed above focus on empirically identifying the key strategies adopted by decision-makers. To our knowledge, ours is the first study that uses the strategy method to compare group and individual choices of strategies and their propensity to experiment with different strategies in an indefinitely repeated game.

\section{Experimental Design}

Each laboratory session employed 24 subjects. In the individual treatment these subjects were subdivided into three groups of 8 subjects, and these 8 subjects interacted independently across all supergames. In the group treatment the 24 subjects were randomly assigned to 3-person groups. Since in the field repeated interactions involving groups quite often involve groups consisting of the same individuals, group composition remained fixed throughout a session. This is standard in the experimental literature studying group behavior (e.g., see Bornstein et al. (2008), Abbink et al. (2010) and other studies cited in footnote 7). These 8 groups interacted in exactly the same way as the 8 individuals, except that they communicated anonymously through computerized chat windows before making every decision. Therefore, in both treatments 8 decision-makers (hereafter DMs) either individuals or groups, played a series of indefinitely repeated, noisy prisoner's dilemma (PD) games. Six independent sets of 8 individuals (48 subjects total) participated in the individual treatment, and four independent sets of 8, 3-person groups (96 subjects total) participated in the group treatment. We also included 64 subjects in a noise-free control condition, summarized in the final subsection of the results section.

In each stage game DMs played the noisy PD with payoffs shown in Table 1a. Table $1 \mathrm{~b}$ displays expected payoffs based on the 1/8 likelihood, drawn iid for each decision, that a choice would be switched to the alternative action. ${ }^{6}$ These payoffs correspond to the highest benefit/cost ratio (4.0) studied in Fudenberg et al. (2012) (hereafter FRD), which they found generated similar cooperation rates as more moderate benefit/cost ratios. Similar to their instructions the game was

\footnotetext{
${ }^{6}$ In contrast to the noisy PD considered in this paper and by Fudenberg et al. (2012) and Aoyagi et al. (2018), in which a DM does not observe the action chosen by the opponent, Gong et al. (2009) and Xiao and Kunreuther (2016) study finitely repeated stochastic PD, in which the actions chosen by the two DMs are perfectly observable to both DMs, but the payoff of each is determined stochastically by the chosen actions. Besides the differences in the structure of uncertainty and their interest in finitely repeated instead of infinitely repeated games, these studies also have objectives that differ from ours, and they do not focus on explicitly identifying the repeated game strategies chosen. Gong et al. (2009) compare behavior of groups to individuals in the finitely repeated stochastic PD, while Xiao and Kunreuther (2016) study the effects of allowing for different forms of punishment in the finitely repeated stochastic PD.
} 
framed without a payoff matrix and without reference to cooperation and defection. Instead, the instructions (shown in Appendix A) simply described the payoff implications of the two action choices. $^{7}$

\section{Table 1: Stage Game Payoffs (panel a) and Expected Payoffs (panel b)}

(a)

\begin{tabular}{l|c|c|}
\multirow{2}{*}{ Cooperate } & Cooperate & Defect \\
\cline { 2 - 3 } Cefect & 6,6 & $-2,8$ \\
\hline \multirow{2}{*}{ Def } & $8,-2$ & 0,0 \\
\cline { 2 - 3 }
\end{tabular}

(b)

\begin{tabular}{l|c|c|}
\multicolumn{1}{c}{} & \multicolumn{1}{c|}{ Cooperate } & Defect \\
\cline { 2 - 3 } Cooperate & $5.25,5.25$ & $-0.75,6.75$ \\
\hline Defect & $6.75,-0.75$ & $0.75,0.75$ \\
\cline { 2 - 3 } & &
\end{tabular}

Each session was divided into three main parts, with a total of 14 repeated games (framed as "interactions"), played twice with each of the 7 other DMs in the session. In the first 4 supergames ("Part I") subjects played the repeated game out round-by-round, in the standard "direct response" method in which the counterparty's action (but not intended choice) was revealed at the end of each round. DMs decided whether to cooperate or defect each round after receiving this feedback. The purpose of these initial games was to familiarize subjects with this repeated game in a more natural presentation. Since the direct response method games always precede the strategy method games (described next) our experiment is not designed to compare behavior in the two elicitation methods (Cason and Mui, 1998; Brandts and Charness, 2000, 2011). This is because the response method is confounded with ordering and learning. We do not include much analysis of the data from these Part I supergames because of this confound, and because this initial play likely reflects early learning that is unrelated to our main research questions.

After Part I was completed instructions were distributed for Part II. Part II lasted for 3 more supergames and DMs chose from a menu of 20 different "plans" that describe different repeated game strategies. ${ }^{8}$ Table 2 describes the 20 plans. The name and abbreviations of the plans (column

\footnotetext{
${ }^{7}$ For example, "If you choose A and the other person chooses A, both you and the other person would get +6 units. If you choose A and the other person chooses B, you would get -2 units, and they would get +8 units." etc.

${ }^{8}$ Equilibrium models of repeated games assume that decision-makers choose and commit to a repeated game strategy in the beginning of a supergame. Therefore, compared to the direct response method, the strategy method implements an environment that is closer to the assumption of theoretical models. Embrey et al.'s (2016) study of an indefinitely repeated $4 \times 4$ games found that overall behavior is similar in the strategy method and the direct response method.
} 
1) were not shown to subjects. A detailed description of each strategy is shown in the Instructions in the Appendix. Hereafter we often refer to the strategies by their abbreviations, for example, Always Defect will be ALLD. Obviously not all strategies can be contained in a finite list, and we sought to strike a balance between comprehensiveness and parsimony with this list of 20 alternatives. As noted earlier, the strategies were drawn from the full list considered by FRD, which they constructed by augmenting strategies that are important in the theoretical literature with suggestions provided by their subjects in a post-experimental survey. Notably, none of these strategies condition on intended rather than implemented actions, which is clearly a restriction. Our analysis of the chat communications indicates that in about one-sixth of the chats subjects refer to the fact that action choices can be switched from the intended action, but during the direct response supergames their discussions did not indicate a strong focus on their past intensions as distinct from past actions.

After subjects selected their plans, the supergame was "played out" round by round as specified in the plan, and subjects saw the results of each round and had to click through to continue on to the next round. As in the direct response supergames 1-4, during these strategy method supergames subjects observed in each round only the action and not the intended choice of their counterpart's plan, or their counterpart's plan choice. ${ }^{9}$ A scrollable round-by-round history was always displayed on screen, showing every previous action by both DMs (and own intended choice), both DMs' earnings, and own cumulative earnings.

For the 7 supergames of Parts I and II, DMs played one repeated game with each of the other DMs (i.e., perfect strangers matching). Then short instructions were distributed for Part III, which consisted of another set of 7 supergames using the strategy method, again with exactly one additional play against each of the other 7 DMs. This matching procedure was emphasized in the instructions. ${ }^{10}$

\footnotetext{
${ }^{9}$ Embrey et al. (2016, forthcoming) also allow subjects to observe in each round the action chosen by their opponent but not the opponent's chosen strategy in their indefinitely repeated oligopoly games.

${ }^{10}$ Abusing terminology, these two sets of 7 supergames were conducted over two sets of perfect strangers matching. We wanted to include more than 7 supergames to be more consistent with the FRD design, which featured roughly 11 supergames per session on average. While conducting all supergames using perfect stranger matching would be preferable this was infeasible for the group treatment given the lab capacity constraints. At least 45 computer stations would be required for 14 perfect stranger supergames played by three-person groups. Since all subjects remain anonymous throughout their session it is impossible for them to recognize when they may be interacting with the same counterpart during supergames $8-14$, so we believe this second match is unlikely to affect the results.
} 
Table 2: Available Strategy Choices (Detailed descriptions are in the Instructions in the Appendix)

\begin{tabular}{lc}
\hline \hline Name (abbreviation) & Plan Number \\
\hline Always cooperate (AllC) & 1 \\
Tit-for-tat (TFT) & 2 \\
Tit-for-2-tats (TF2T) & 3 \\
Tit-for-3-tats (TF2T) & 4 \\
2-tits-for-tat (2TFT) & 5 \\
2-tits-for-2-tats (2TF2T) & 6 \\
T2 (T2) & 7 \\
Win-Stay-Lose-Shift (PTFT) & 8 \\
WSLS with 2 rounds punish (2PTFT) & 9 \\
Grim Trigger (Grim) & 10 \\
Lenient Grim (Grim2) & 11 \\
More Lenient Grim (Grim3) & 12 \\
False cooperator (C-to-ALLD) & 13 \\
Always defect (ALLD) & 14 \\
Exploitive tit-for-tat (D-TFT) & 15 \\
Exploitive tit-for-2-tats (D-TF2T) & 16 \\
Exploitive tit-for-3-tats (D-TF3T) & 17 \\
Exploitive Grim2 (D-Grim2) & 18 \\
Exploitive Grim3 (D-Grim3) & 19 \\
Alternator (DC-Alt) & 20 \\
\hline \hline
\end{tabular}

Each supergame terminated after every round with a probability $1 / 8$, so the expected length was 8 rounds. Following a standard practice in this literature, the length of each supergame was determined in advance and the same sequence of supergame lengths was used across all sessions and treatments. This is because the length of supergames has been shown to impact behavior (Engle-Warnick and Slonim, 2006; Dal Bó and Fréchette, 2011), and by using the same pattern of lengths this influence is held constant across sessions and treatments. The 14 supergames varied in length from 1 to 31 rounds, with an average of 7.57 rounds. ${ }^{11}$

As mentioned above, groups made each decision by majority vote following a period of computerized, anonymous chat. Before the chat window was open, each member of the 3-person group made a nonbinding "proposal" for a choice -- either cooperate or defect during supergames $1-4$, or a repeated game strategy plan number for supergames $5-14$. The subjects then made binding

\footnotetext{
${ }^{11}$ The drawn lengths were $4,2,9,5,14,10,3,2,31,9,3,1,5$ and 8 rounds.
} 
votes for their group's choice when the chat time was completed. ${ }^{12}$ Votes in the strategy method supergames were unanimous 98 percent of the time, and the stated tie-breaking rule (where one of the three voted strategies was selected at random) was never needed. All six times the vote was not unanimous one strategy was favored by two of the three group members.

After all 14 supergames were completed, subjects completed the standard three-question Cognitive Reflection Test (Frederick, 2005), receiving US\$1.00 for each correct answer. Subjects answered these questions individually even if they participated in the group treatment. Subjects also completed an individual lottery investment task (Gneezy and Potters, 1997) to reveal their willingness to take small financial risks, and then answered a post-experiment questionnaire to report their motivations for their strategy choices and some demographic descriptors.

All sessions were conducted at Purdue University using zTree (Fischbacher, 2007). Subjects were recruited broadly from the undergraduate student population using ORSEE (Greiner, 2015). Sessions on average lasted about 90 minutes for the individual treatment and 2 hours for the group treatment and the 208 unique subjects earned US\$34.67 on average with an interquartile range of $[\$ 26.50, \$ 42.50]$.

In the strategy method supergames, the two DMs play a 20x20 game. Table 3 reports results from simulations we conducted to determine the (pure-strategy) Nash equilibria in this game. As FRD observes, since each DM's action is switched with a positive probability regardless of the actions played, every information set is reached with positive probability. Hence, Nash equilibrium implies sequential rationality. Thus in the infinitely repeated noisy PD every Nash equilibrium is a sequential equilibrium, and "every pure-strategy Nash equilibrium is equivalent to a perfect public equilibrium" (FRD, p. 725). Table 3 shows that this game has eight equilibria: (ALLD, ALLD), (Grim, Grim), (Grim 2, Grim 2), (PTFT, PTFT), (2PTFT, 2PTFT), (D-Grim 2, D-Grim 2), (D-Grim 3, D-Grim 3), (T2, T2). Three additional strategies (ALLC, TF3T and ALT) are best responses to at least one pure strategy, and most of the remaining strategies are rationalizable because they are a best response to some belief about the distribution of strategies chosen by others. The "Average" column shows the expected performance of each strategy against the uniform prior of an equal population of all strategies. Best-performing strategies include those that are slow to

\footnotetext{
12 The chat time was 45 seconds before each round of the direct response supergames 1-4. For the strategy method supergames, the chat time was 6 minutes before supergame 5,5 minutes before supergame 6,4 minutes before supergame 7 , and 3 minutes before supergames $8-14$.
} 
anger, and fast to forgive (Grim3, 2TF2T, TF2T, TF3T, etc.), while ALLD performs the worst. The same broad conclusion holds when focusing on only those strategies that are chosen in the experiment most frequently, although the individual ranking varies somewhat.

Table 3: Expected Payoff Matrix (5000 simulations)

\begin{tabular}{|c|c|c|c|c|c|c|c|c|c|c|c|c|c|c|c|c|c|c|c|c|c|}
\hline & rage & IC & $=T$ & $=2 T$ & $=3 T$ & $\mathrm{FT}$ & $\mathrm{F} 2 \mathrm{~T}$ & irim & im2 & im3 & ID & TFT & TFT & TF2 & $11=$ & D-Gr & & & T2 & & \\
\hline AllC & 7.71 & 16 & .66 & 1.44 & 41.95 & & 1.95 & 1.96 & .86 & .59 & 06 & .78 & 76 & 648 & 15.78 & & & 5.92 & 3.72 & 0.05 & 165 \\
\hline$T F T$ & 7.53 & 33 & .69 & & 3.21 & 84 & 9.2 & 3.52 & & 6.53 & 17 & .68 & .96 & & & & & 01 & & 8.2 & 9 \\
\hline TF2 & 3.81 & .25 & 58 & & $7=$ & & 0.75 & & & .05 & & 53 & & & & & & .68 & & 32 & \\
\hline TF3T & 3.80 & 06 & 6.7 & & 1.93 & 59 & .92 & 16.01 & & .92 & & .47 & .65 & 35.59 & & & & 9.72 & 7.58 & 86 & 6.8 \\
\hline 2TFT & 17 & 1.4 & 26.1 & & & & .93 & & & 88 & & .86 & & & & & & 1.99 & & & \\
\hline $2 T F 2$ & .82 & 43 & 1.79 & & & & 40 & & & & & 1.69 & & & & & & 43 & & 99 & 7.8 \\
\hline Grim & .27 & 47 & 74 & & & & 3.37 & 19. & & .29 & & .66 & 3.59 & .96 & & & & 61 & 8.99 & 1.3 & 6.65 \\
\hline Grim2 & 7.80 & 68 & .21 & 3 & & .25 & 45 & 18. & 14 & .73 & 79 & .04 & & .31 & 2.51 & & & 46 & 8.21 & 83 & 1.5 \\
\hline Grim3 & .92 & .56 & 48 & 0.01 & 1 & & 39.43 & 17 & 4 & .31 & & 6.6 & 9.67 & 3.93 & 35.14 & & & 4.18 & 7.47 & 5.57 & 20.39 \\
\hline $\mathrm{Al}$ & .95 & .12 & 7.2 & 2 & 2.55 & 1 & 2.93 & & & .74 & & .31 & 2.04 & .96 & .53 & & & 1.76 & 5.55 & 12.06 & 8.3 \\
\hline D-TFT & 5.17 & .94 & 26.21 & 4 & 8 & 81 & 8.65 & 11.11 & & .21 & & .62 & & & 3.66 & & & 08 & & .7 & 3.45 \\
\hline PTFT & 5.17 & 5.7 & 9.6 & 66 & 40.71 & 4 & 5.08 & 16. & 88 & 7.55 & .34 & 24 & 5.31 & 0.57 & 35.13 & & & 54 & 3.98 & 5.1 & 2.18 \\
\hline D-TF2T & 27.60 & .74 & .99 & 4 & & 15 & 1.61 & 9. & & & & 39 & .98 & .77 & & & & 29 & .46 & 68 & 3.84 \\
\hline D-TF3T & 27.86 & .68 & 4.19 & 42.62 & 3.57 & 24.96 & 41.93 & 7.8 & \begin{tabular}{|l|}
31.34 \\
\end{tabular} & 40 & 0. & 7.7 & 1.72 & 36.49 & 37.63 & & .47 & 4.08 & 4.73 & .27 & 8.34 \\
\hline D-Grim2 & 26.20 & .16 & .31 & 37.5 & & & 36.04 & 11.72 & 32.48 & 3 & & 5.04 & 29.69 & 97 & 32 & .62 & & 2.09 & 78 & 0.3 & 3.02 \\
\hline D-Grim 3 & 27.75 & .44 & .87 & 39 & 42.41 & .06 & 39.39 & 10.34 & 3 & .06 & 2.91 & 3.51 & 27.34 & .8 & .14 & & & 9.53 & 3.83 & 3.9 & 2.09 \\
\hline $2 \mathrm{P}$ & 25.20 & .72 & 27.27 & 34.5 & 38. & 3 & 0.77 & 1 & & 38 & & 3.23 & 34.18 & 7.38 & 49 & 0.72 & 67 & .61 & 1.18 & 84 & 5.05 \\
\hline T2 & 25.79 & 39 & 29.4 & 3 & & .05 & 2.82 & 17. & & 26 & 16 & .84 & .88 & 31.71 & 02 & 44 & 52 & 3.43 & 2.06 & 62 & 2.56 \\
\hline All & 2.60 & & 6 & & & & 24.63 & 15.04 & & 25.96 & & 3.57 & 32.59 & .4 & .23 & & 91 & 5.06 & 6.81 & 10.46 & .02 \\
\hline ALT & 37 & 3.44 & 6.42 & 44.18 & 75 & 11.2 & 2.88 & 7.56 & 99 & & & & t. & & 0.42 & 1.93 & & 13.9 & 4.1 & 31 & 22. \\
\hline
\end{tabular}

Notes: Best-responses highlighted. Eight equilibria, with All-D the worst and PTFT (WSLS) the best, are highlighted in bold. The average column indicates the expected performance against an equal population of all strategies (i.e., a uniform prior). The ten best-performing strategies highlighted with italics. Best-performing strategies include those that are slow to anger, and fast to forgive (Grim3, 2TF2T, TF2T, TF3T, etc.). ALLD is the worst.

\section{Results}

We begin the results presentation in Section 3.1 with an overview of the most common strategy choices. Section 3.2 then compares strategies chosen by groups and individuals after aggregating the strategy choices into various types. Section 3.3 documents how DMs experiment with different strategy choices, and shows that the experimentation rate declines over time. Section 3.4 compares the overall cooperation rate between groups and individuals and Section 3.5 examines the popularity of the ALLD strategy. The final subsection summarizes results form a control condition with perfect monitoring, where the action implementation noise is eliminated.

\subsection{Most Common Strategy Choices}

Subjects in the noisy PD selected repeated game strategies a total of 800 times across all 10 strategy method supergames (480 in the individual treatment and 320 in the group treatment). All 20 strategies were chosen at least once in both treatments, several exactly once. Figure 1 displays the 
most common strategy choices, including all strategies that were chosen at least 5 percent of the time either by groups or individuals. These displayed strategies represent more than 80 percent of

Figure 1: Most Frequent Strategy Choices
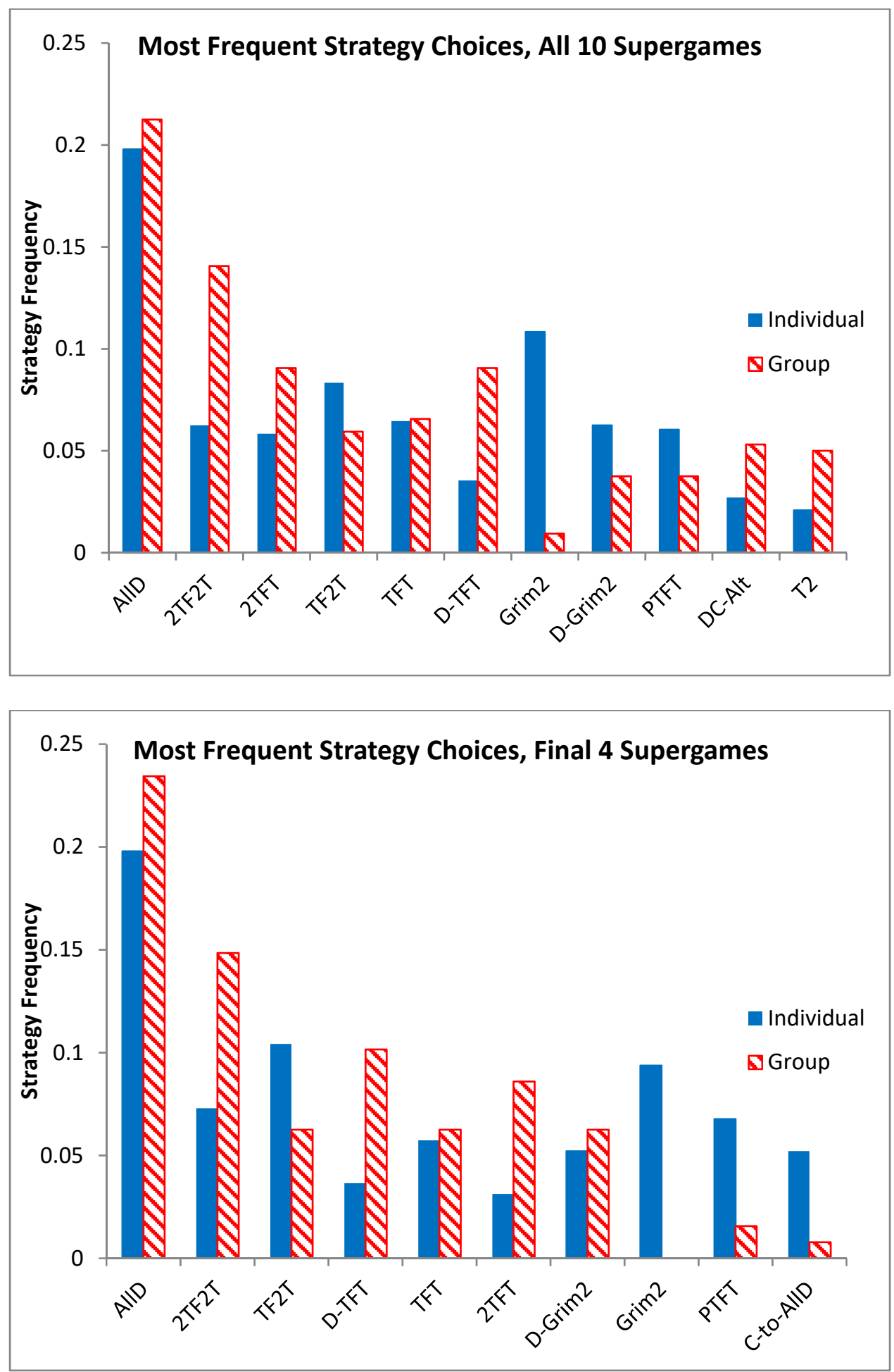
all strategies selected in the experiment (646 out of 800 choices). The overall distributions are not substantially different between the upper (all strategy method supergames) and bottom (final four supergames), and our statistical comparison also does not detect important differences in strategy choices between early and late supergames.

The most common strategy is ALLD. This strategy performs poorly and returns low expected and actual average payoffs, but it was also the most common strategy identified by FRD. Section 3.5 below explores the frequent choice of this strategy in more detail. Subjects also often chose different versions of the tit-for-tat strategy, with the lenient and forgiving two-tits-for-twotats (abbreviated 2TF2T) being the most common choice for groups. Some lenient versions of grim trigger are also common, such as Grim2 that is not triggered unless the counterparty defects for two consecutive periods.

\subsection{Comparison of Group and Individual Strategy Choices}

Our first research question concerns how groups' strategy choices compare to individuals', with particular attention to whether groups also employ cooperative, lenient and forgiving strategies in this noisy repeated PD. Following FRD we designate strategies as lenient if they do not switch to defection until the counterparty defects for two or more rounds. This includes strategies such as TF2T and Grim2. We designate strategies as forgiving if they can switch back to a cooperative phase, so this includes all of the TFT variants and excludes all versions of Grim. Of course, these classifications are not mutually exclusive; as noted earlier, for example, 2TF2T is both lenient and forgiving.

Many lenient and forgiving strategies begin by cooperating in the first round, but others begin by defecting in the first round before (possibly) switching to cooperation. We follow FRD and refer to these defect-first strategies, such as D-Grim2 and D-TFT as "exploitive" lenient or forgiving strategies. We consider other groupings as well in the summary shown in Table 4. This includes mutually exclusive categories based on the type of repeated game logic they employ, such as those based on tit-for-tat and those that employ grim triggers. The cooperative strategies designated at the top of the table include all strategies other than All Defect and C-to-All Defect that never choose to cooperate after the first round.

Table 4 summarizes the frequency of the different strategy types in the two treatments in two columns. A statistical comparison of the treatments must account for the panel nature of the dataset, as well as a time trend, which we do here using a random effects logit regression with 
random decision-maker effects and robust standard errors clustered on sessions. ${ }^{13}$ From such tests we conclude the following:

Finding 1: Compared to the individual treatment studied in previous experiments, groups employ significantly more forgiving strategies, especially those that are exploitive, more tit-for-tat strategies, and fewer grim strategies. Groups also select equilibrium strategies less often that do individuals. Groups, however, also behave similarly to individuals in many dimensions.

Although Finding 1 indicates some different strategy choices when groups rather than individuals play the noisy, repeated PD, overall groups do not select radically different strategies than individuals. They are equally likely to choose strategies that involve some cooperation (about 77 percent), or some exploitation (about 18 percent), and they do not employ more complex strategies that are based on greater memory length. Although individuals on average more frequently choose lenient strategies, groups do not differ significantly from individuals on these types of strategies. Our content analysis of the group chats reveals a direct association between these lenient strategy choices and subjects' concerns about the noisy implementation of stage game actions. ${ }^{14}$ Groups who chose lenient strategies discussed "how choices can be switched to the other action" (Kappa reliability 0.701 ) in $23 \%$ of the preceding chat rooms, compared to $16 \%$ for the groups who did not choose lenient strategies.

In their recent studies on the finitely repeated PD using the direct response method, Gong et al. (2009) (who study the stochastic PD) and Kagel and McGee (2016) (who study the deterministic PD) find that groups cooperate more than individuals. This finding contrasts with the conclusion that groups cooperate less than individuals in the finitely repeated deterministic PD in the "group discontinuity" literature in psychology (Wildschut et al., 2003, Wildschut and Insko, 2007). Kagel and McGee (2016) point out that the studies in psychology typically involved a single repeated game between two DMs, while in their study, like in most economic experiments, DMs plays a number of repeated games and re-matched with other DM following each supergame. This design allows Kagel and McGee (2016) to investigate the question of whether the "discontinuity effect" persists overtime, which has not received much attention in the psychology literature.

\footnotetext{
${ }^{13} \mathrm{We}$ account for the time trend by including the inverse of the strategy method supergame number in the regressions, which allows for trends to be stronger in earlier supergames and weaker in later supergames. This is a common specification for experimental data.

${ }^{14}$ For the content analysis we hired two coders who were unaware of our research questions to code independently all chat room dialogs into 32 possible content categories and subcategories. Coding reliability was assessed using Cohen's Kappa, and we only analyzed categories that reached the "moderate" Kappa agreement threshold of 0.4 or greater.
} 
Similar to Kagel and McGee (2016), Gong et al. (2009) and this study both employ the typical design in economic experiments that allow re-matching and the play of multiple repeated games by DMs. For reasons that we shall explain below, while we find that groups employ significantly more forgiving strategies, more tit-for-tat strategies, and fewer grim strategies than individuals in this indefinitely repeated noisy PD with the strategy method, the cooperation rates between individuals and groups do not differ overall in our experiment.

\section{Table 4: Frequency of Strategy Type Classifications}

\begin{tabular}{|l|l|l|l|}
\hline Strategy Type & $\begin{array}{l}\text { Individual } \\
\text { Treatment }\end{array}$ & $\begin{array}{l}\text { Group } \\
\text { Treatment }\end{array}$ & $p$-value \\
\hline Cooperative & 0.771 & 0.778 & 0.648 \\
\hline Lenient & 0.308 & 0.266 & 0.569 \\
\hline Lenient including Exploitive & 0.446 & 0.356 & 0.229 \\
\hline Forgiving & 0.296 & 0.384 & $0.086^{*}$ \\
\hline Forgiving including Exploitive & 0.373 & 0.525 & $0.021^{* *}$ \\
\hline Exploitive & 0.173 & 0.181 & 0.566 \\
\hline Some Tit-for-Tat & 0.450 & 0.613 & $0.008^{* * *}$ \\
\hline Some Grim & 0.269 & 0.109 & $0.013^{* *}$ \\
\hline Equilibrium & 0.542 & 0.384 & $0.002^{* * *}$ \\
\hline Memory $>1$ & 0.521 & 0.497 & 0.641 \\
\hline Change Strategy & 0.248 & 0.208 & 0.645 \\
\hline
\end{tabular}

Note: $p$-values based on a logit regression that controls for a time trend, with subject random effects and clustering by session (all twotailed and based on all ten strategy method supergames).

$*, * *$ and $* * *$ highlight treatment differences at the $10-, 5$ - and 1percent significance levels.

\subsection{Changes in Strategy Choices across Supergames}

A second key research question concerns how frequently groups experiment with different strategies compared to individuals. A distinct advantage of eliciting strategies directly rather than inferring them from round-by-round actions is that the data precisely reveal the stability of strategy choices and changes in strategy choices across supergames. By contrast, when employing the Strategy Frequency Estimation Method (hereafter SFEM) developed in Dal Bó and Fréchette, 2011) researchers typically assume that strategy choices remain unchanged across supergames. Our data allows us to evaluate the accuracy of this assumption, albeit using a different (strategy) choice elicitation method that could affect the frequency that new strategies are adopted. 
Figure 2: Frequency of Strategy Changes Across Supergames

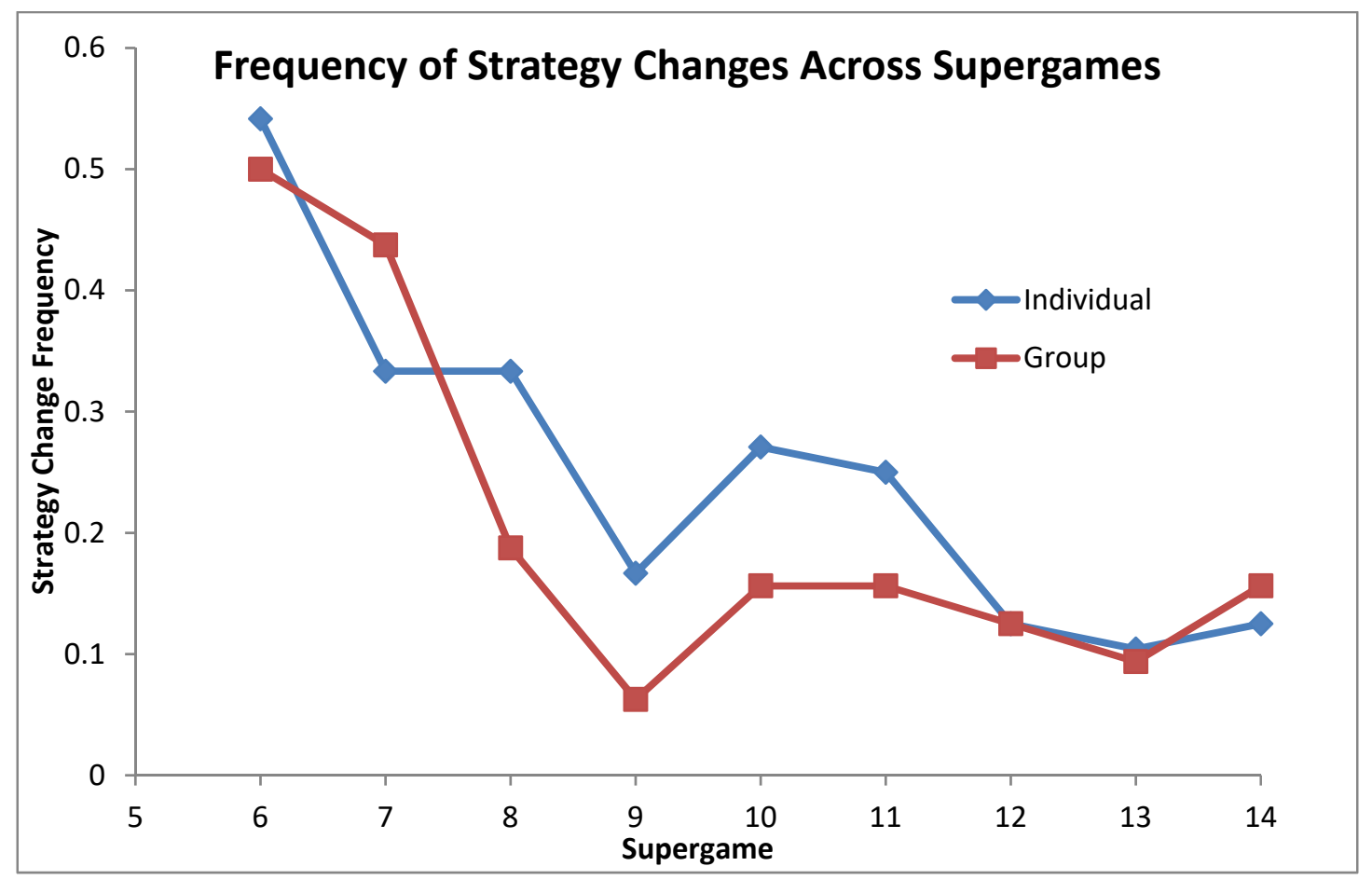

The final row of Table 4 indicates that DMs changed strategies in consecutive supergames about 21 to 25 percent of the time overall, and this frequency is not significantly different between groups and individuals. Figure 2 illustrates that these strategy choice changes become less frequent in the later supergames, and that this time trend is also similar across treatments. (This time trend is also highly significant in the random effects logit regression, with a $p$-value $<0.01$.) For both groups and individuals, strategy changes are significantly more common when the current strategy results in low per-round payoffs (random effects logit model $p$-values $<0.01$ ).

Previous applications of SFEM often focus on later supergames, after subjects have become very familiar with the game so that their strategies and actions become more stable. For example, FRD focus on the final four supergames of their sessions. The SFEM assumption of stable strategy choices is considerably more accurate in the last four supergames for our data. In particular, strategy changes occur across these final 4 supergames only 12 percent of the time. During these final 4 supergames, 72 percent of the groups and 79 percent of the individuals never change strategies. Thus, our direct evidence that strategy choices tend to stabilize for the later supergames in the indefinitely repeated noisy PD and Embrey et al. (2016)'s evidence that strategy 
choices also stabilize for the later supergames in their indefinitely repeated oligopoly games indicate that the standard SFEM assumption of stable choices is relatively well satisfied, at least when focusing on the later supergames in a session.

Finding 2: Groups and individuals experiment infrequently with different strategies, especially in later supergames, and they change strategy choices at similar rates.

A few systematic patterns exist in the plan changes. For instance, in the group treatment the rate that decision-makers change from a lenient plan to a non-lenient plan tends to be greater than the overall rate that non-lenient plans are chosen. Some groups employed but then abandoned lenient strategies when these were taken advantage of by their counterparty, as illustrated by the following chat room excerpt:

member 2: i dont like 11[Note: Plan 11 is Grim2]

member 2: it made us lose so many times

$\ldots$

member 1: $1 / 8$ killed us :(

member 2: and i dont w2ant to give them 2 rounds

member 2: no it actually saved us

member 1: i guess

member 1: but it made them a bunch of extra money too haha

member 2: yeah

member 2: i dont want other team to get more than 1 round of benefit

(session 101, group 1, supergame 6)

Another pattern in the plan changes is that individual DMs who switched from a forgiving plan were more likely to stay with another forgiving plan rather than switch to a non-forgiving plan. As documented in the previous subsection, however, they did not adopt forgiving plans as often as did groups.

Table 5 summarizes the transitions between broad types of strategies to illustrate more systematically how groups (panel a) and individuals (panel b) move within the different strategy classifications. Clearly more transitions align on the diagonal, indicating stability of strategy choices within these broad classifications. A modest degree of movement is evident between the popular Tit-for-Tat and Grim strategy types for the individual treatment (16 and 13 transitions). 
Overall, however, for the most common types (Tit-for-Tat, Grim, and Always Defect), typically 80 to 90 percent of the strategy choice transitions remain within the same broad classification.

Table 5: Transitions between Broad and Mutually-Exclusive Strategy Types

Panel a: Groups

New Strategy Choice (supergame $t$ )

\begin{tabular}{|c|c|c|c|c|c|c|}
\hline & & $\begin{array}{l}\text { Always } \\
\text { Cooperate }\end{array}$ & $\begin{array}{l}\text { Some Tit- } \\
\text { for-Tat }\end{array}$ & Some Grim & $\begin{array}{c}\text { Always } \\
\text { Defect }\end{array}$ & Alternate \\
\hline \multirow{5}{*}{$\begin{array}{l}\text { Previous } \\
\text { Strategy } \\
\text { Choice } \\
\text { (supergame } t-1 \text { ) }\end{array}$} & \multirow{5}{*}{$\begin{array}{c}\text { Always Cooperate } \\
\text { Some Tit-for-Tat } \\
\text { Some Grim } \\
\text { Always Defect } \\
\text { Alternate }\end{array}$} & & & & & \\
\hline & & $1(0.6 \%)$ & $163(92.1 \%)$ & $4(2.3 \%)$ & $7(4.0 \%)$ & $2(1.1 \%)$ \\
\hline & & & $4(12.9 \%)$ & $23(74.2 \%)$ & $3(9.7 \%)$ & $1(3.2 \%)$ \\
\hline & & & $6(9.4 \%)$ & $2(3.1 \%)$ & $56(87.5 \%)$ & \\
\hline & & & $1(6.3 \%)$ & $2(12.5 \%)$ & & $13(81.3 \%)$ \\
\hline
\end{tabular}

Panel a: Individuals

New Strategy Choice (supergame $t$ )

\begin{tabular}{|c|c|c|c|c|c|c|}
\hline \multirow{6}{*}{$\begin{array}{l}\text { Previous } \\
\text { Strategy } \\
\text { Choice } \\
\text { (supergame } t-1 \text { ) }\end{array}$} & \multirow{6}{*}{$\begin{array}{c}\text { Always Cooperate } \\
\text { Some Tit-for-Tat } \\
\text { Some Grim } \\
\text { Always Defect } \\
\text { Alternate }\end{array}$} & $\begin{array}{c}\text { Always } \\
\text { Cooperate }\end{array}$ & $\begin{array}{l}\text { Some Tit- } \\
\text { for-Tat }\end{array}$ & Some Grim & $\begin{array}{c}\text { Always } \\
\text { Defect }\end{array}$ & Alternate \\
\hline & & $9(81.8 \%)$ & $1(9.1 \%)$ & $1(9.1 \%)$ & & \\
\hline & & & $171(88.1 \%)$ & $13(6.7 \%)$ & $7(3.6 \%)$ & $3(1.5 \%)$ \\
\hline & & $1(0.8 \%)$ & $16(13.4 \%)$ & $96(80.7 \%)$ & $4(3.4 \%)$ & $2(1.7 \%)$ \\
\hline & & & $4(4.1 \%)$ & $4(4.1 \%)$ & $88(89.8 \%)$ & $2(2.0 \%)$ \\
\hline & & & $3(30.0 \%)$ & $3(30.0 \%)$ & & $4(40.0 \%)$ \\
\hline
\end{tabular}

\subsection{Overall Cooperation Rate for Groups and Individuals}

Motivated by the group discontinuity literature, our third research question concerns the overall amount of cooperation exhibited by groups relative to individuals. This reveals that a simple comparison of the average degree of cooperation can miss important underlying differences and heterogeneity of behavior and adopted strategies, documented earlier through differences in strategies chosen by groups and individual DMs (Finding 1). In particular, we conclude the following:

Finding 3: The average rate of cooperation does not differ significantly between groups and individuals, either in the first rounds of each supergame, across all rounds, in the direct response method condition, or in later strategy method supergames. 
This finding is illustrated in Figure 3. As documented in earlier studies such as FRD and Aoyagi et al. (2018), the cooperation rate in the first round of each supergame tends to exceed the cooperation rate across all rounds. Cooperation rates also tend to be higher in the strategy method supergames than in the direct response method supergames, but this increase in cooperation across supergames (particularly for first round cooperation) is also seen when all supergames employ the direct response method (cf. Figure 2 of FRD). Importantly, the patterns are similar across the group and individual treatments, and our statistical tests never detect any treatment differences. ${ }^{15}$ For every comparison the p-value exceeds 0.66 .

Figure 3: Overall Cooperation Rate Across Treatments

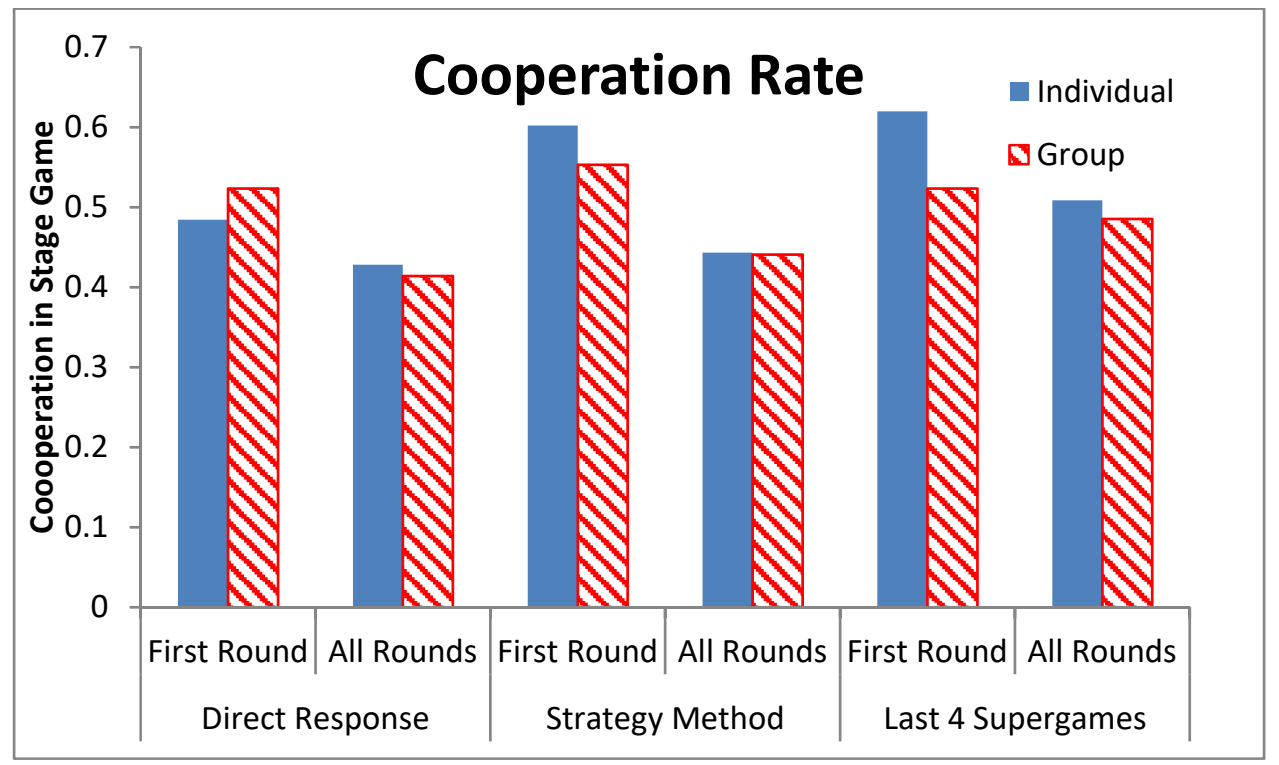

Do findings 1 and 3 conflict? How can strategy choices differ but cooperation rates not differ across treatments? A closer examination of the specific strategy choices within the broad strategy types shows that the findings are not actually in conflict. This is because strategies within each type have different realized cooperation rates and they are chosen with differing frequency by groups and individuals. For example, although groups employ forgiving and tit-for-tat strategies

\footnotetext{
${ }^{15}$ For these tests we employ logit panel regressions with the binary choice to cooperate in a round as the dependent variable. They account for a time trend using the inverse of the supergame number, and employ random decisionmaker effects and robust standard errors clustered on sessions.
} 
more than individuals, as Figure 1 illustrates groups more frequently choose 2-tits-for-2-tats and less frequently choose tit-for-2-tats. The two rounds of punishment in 2-tits-for-2-tats leads this strategy to have a lower rate of cooperation (about 60 percent) than tit-for-2-tats (about 72 percent), based on the actual pairing of strategies realized in the experiment. Consequently, the greater frequency of forgiving and tit-for-tat strategy choices by groups does not translate into a greater overall cooperation rate.

We also considered how the initial choice about whether to cooperate might be related to subject characteristics, prior to any supergame with others in these repeated games. For the individual treatment this is the first round choice of whether to cooperate in the first supergame. For the group treatment this is the first proposal of whether or not to cooperate in the first round of the first supergame. This initial propensity to cooperate is not significantly related to the subjects' gender, risk preferences, or score on the Cognitive Reflection Test (CRT).

\subsection{The Popularity of Always Defect}

We conjectured that group play may diminish the popularity of ALLD. This conjecture, however, is not supported by the data. In spite of the relatively high cooperation rate in this repeated noisy PD with its relatively strong returns to cooperation, in both the individual and group treatments, ALLD is the most popular strategy and its popularity does not decrease over time (Figure 1). While this strategy performs poorly when others employ conditionally cooperative strategies, it is of course an equilibrium strategy and it is a best response to a sufficiently strong belief that others are also choosing this strategy (or any other history independent strategy). FRD also find that ALLD is the most common strategy choice in their experiment when using the same $1 / 8$ error rate that we also employ. They attribute this to the complexity of the environment making it difficult to learn the optimal response. Aoyagi et al. (2018) also estimate that ALLD is the first or second most common strategy employed in all treatments of their experiment. ${ }^{16}$

ALLD is a best response to ALLD or any other history-independent strategy. It is not a best response, however, to the strategies that are selected. Based on our simulation interacting the 20 strategies shown earlier in Table 3, ALLD performs worst when its expected value is calculated

\footnotetext{
${ }^{16}$ We estimated the strategy choices based on the direct response method supergames 1-4 using the Strategy Frequency Estimation Method and also determined that always defect was employed most frequently in these early supergames. In particular, this method estimates that 31 percent of the group strategies and 44 percent of the individual strategies are always defect. Similar to Figure 1, the tit-for-tat strategies were also commonly estimated, combining for 26 percent of the group strategies and 38 percent of the individual strategies.
} 
using the empirical distribution of strategies actually chosen by our subjects, while slow to anger and fast to forgive strategies (2TF2T, Grim3, TF2T, TF3T, etc.) again perform the best. Figure 4 displays the expected payoff of the "most popular" 10 strategies shown in the lower panel of Figure 1 , based on the population distribution of strategies selected for the final four supergames. Lenient and forgiving strategies do particularly well, and the best performing strategy for the individual treatment is $2 \mathrm{TF} 2 \mathrm{~T}$, and for the group treatment is TF2T. Expected payoffs for ALLD are about 30 percent lower than these best strategies. For the smaller sample size of 10 supergames actually used in the experiment, rather than the 5000 supergames of the simulation, ALLD does better than worst but is still below average, finishing in twelfth place out of 20. Nevertheless, based on realized earnings of each strategy, DMs could earn about 30 percent more by switching from ALLD to a better strategy.

Figure 4: Expected Payoffs for Common Strategies

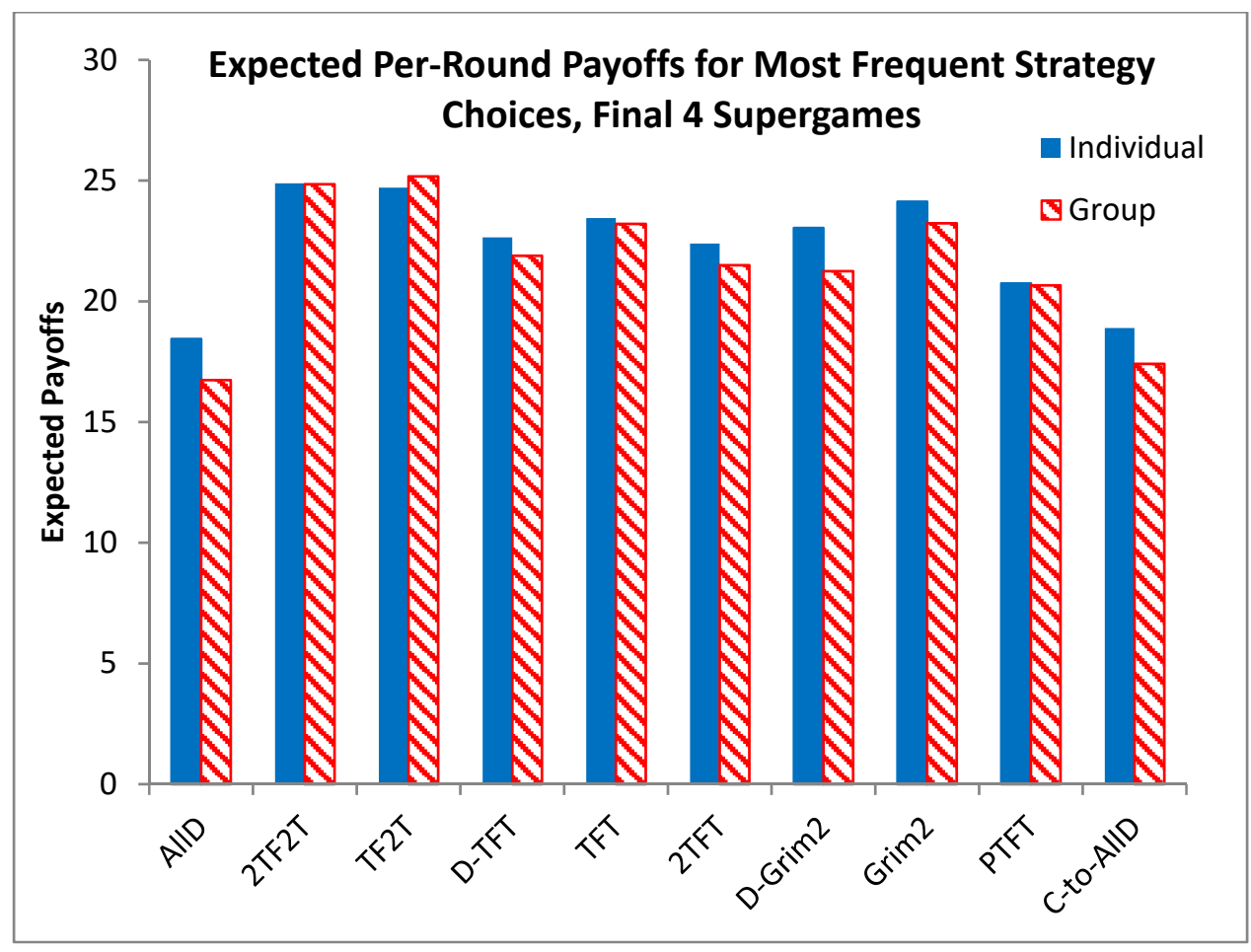

In order to learn the poor relative performance of the ALLD strategy the DMs need to try other, history-dependent strategies. Like FRD, however, we find that a minority of individuals and 
groups persist in choosing this inferior ALLD strategy and seldom try others. Six of the 32 groups are responsible for nearly all of the ALLD strategy choices, and 7 of the 48 individuals are unwavering ALLD players while 4 others choose this strategy exactly 4 times during the 10 supergames. In other words, 13 of the 80 decision-makers ( 16 percent) are responsible for most of the ALLD strategy choices, which represent about 20 percent of all strategy choices.

These 13 DMs who choose ALLD in a majority of the supergames scored significantly worse on the CRT compared to the others who did not choose this strategy as frequently. ${ }^{17}$ For groups we considered the mean CRT score across all 3 group members, and this averaged 1.08 correct answers (out of 3 ) for the majority ALLD groups compared to 1.54 correct for the other groups; this difference is statistically significant based on a logit regression with robust standard errors clustered on sessions (two-tailed $p$-value $<0.05$ ). Maximum and minimum CRT scores within the group are correlated significantly with ALLD choices similar to mean CRT scores. ${ }^{18}$ In the individual treatment the average CRT score was 1.14 for the majority ALLD individuals compared to 1.41 for the others, but this difference is not statistically significant. For the individual treatment women more frequently chose ALLD in the majority of their supergames compared to men, as determined by a logit model that also controls for CRT score (and again with session clustering; two-tailed $p$-value $<0.05$ ). This gender difference is not identified in the group decisionmaking treatment. This greater choice of ALLD for women is consistent with evidence that women tend to more strongly prefer to avoid risk than men (Croson and Gneezy, 2009), since defect choices avoid the risk of the sucker payoff $(-2){ }^{19}$

\footnotetext{
${ }^{17}$ The CRT consists of three questions. As Frederick (2005) explains, for each question, respondents often give an intuitive but erroneous answer quickly, and getting the correct answer requires them to avoid this "impulsive" answer and engage in further reflection to identify the correct answer. A significant percentage of responders, including many from selective colleges in the US, give incorrect impulsive answers frequently. Toplak et al. (2011) argue that CRT measures both a person's cognitive ability and thinking disposition, with the latter including reflectivity and "the tendency to seek alternative solutions" (Toplak et al., 2011, p. 1276). CRT scores are found to be correlated with time preferences, risk attitudes (Frederick, 2005) a large number of decision tasks studied in the literature on heuristic and decision-making (Toplak et al., 2011), and overbidding in contests (Sheremeta, 2016).

${ }^{18}$ The six groups who mostly chose ALLD included one group in which all three members answered all three CRT questions incorrectly, and one group in which one member answered correctly for one question and no other answers for any other group members were correct. A third group had two members with one correct answer and zero correct answers for the third member. For the other three groups at least on member answer all three CRT questions correctly. ${ }^{19}$ This raises the natural question of whether ALLD could be a best response to the population distribution of strategy choices for sufficiently risk averse subjects. This is a difficult question to answer, since considerable payoff variance of a strategy arises from variance in supergame lengths, but we believe that risk aversion is not a good explanation by itself for ALLD strategy choices for a couple of reasons. Based on the realized mean and variance of the payoffs earned for each strategy, several of the strategies with greater average payoffs than ALLD also have a lower variance. We also do not find any significant correlation between the propensities to mostly choose ALLD with the amount invested in our risk assessment task at the end of the experimental sessions. In recent survey articles, Charness and
} 
Based on our content analysis of the group chats, we found a relationship between appeals to game theoretic reasoning and adoption of the ALLD strategy. This is illustrated in the following chat room exchange:

\begin{tabular}{|ll|}
\hline member 1: & why 14? [Note: Plan 14 is ALLD] \\
member 3: & because it is only logical \\
member 3: & always choose B [Note: Action B is Defect] \\
member 3: & why would you want to lose points \\
member 3: & it is simple econ \\
member 1: & kk \\
& $\ldots$ \\
member 3: & just take an intro econ course with blanchard \\
member 3: & $\ldots$ \\
member 2: & gotcha. well either way, always B \\
& (session 101, group 4, supergame 5) \\
\hline
\end{tabular}

Groups who chose the ALLD strategy were about three times more likely to have their chat classified to contain reference to game theory or economic reasoning (Kappa reliability 0.705) compared to groups who chose lenient, forgiving or tit-for-tat strategies.

Groups also more frequently refer to game theory or economic reasoning when they selected strategies that employ grim triggers. ${ }^{20}$ This excerpt contains chat rooms for the same group in consecutive supergames:

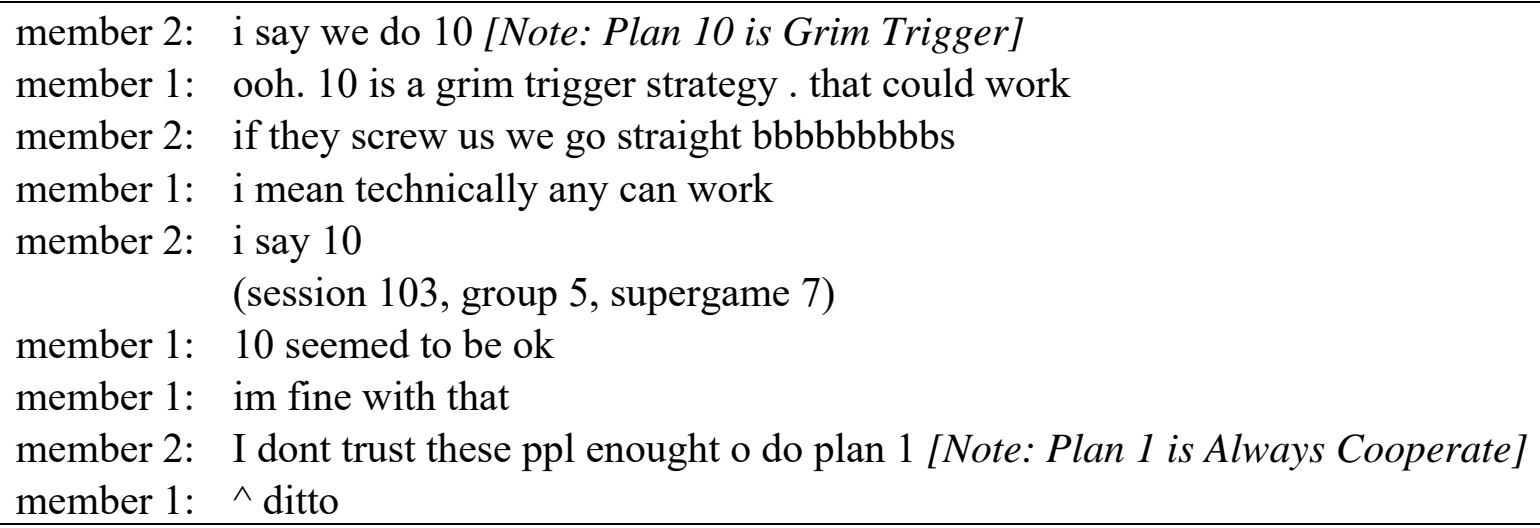

Sutter (2012b, p.3) and Kugler et al. (2012, p. 472) conclude that experimental studies have produced mixed findings on whether groups take on more or less risk than individuals.

${ }^{20}$ The subject pool does not include a substantial fraction of economics students, and only 7 of the 144 subjects in the study were economics majors. Member 1 in the following chat who knows the grim trigger strategy is not an economics major. 


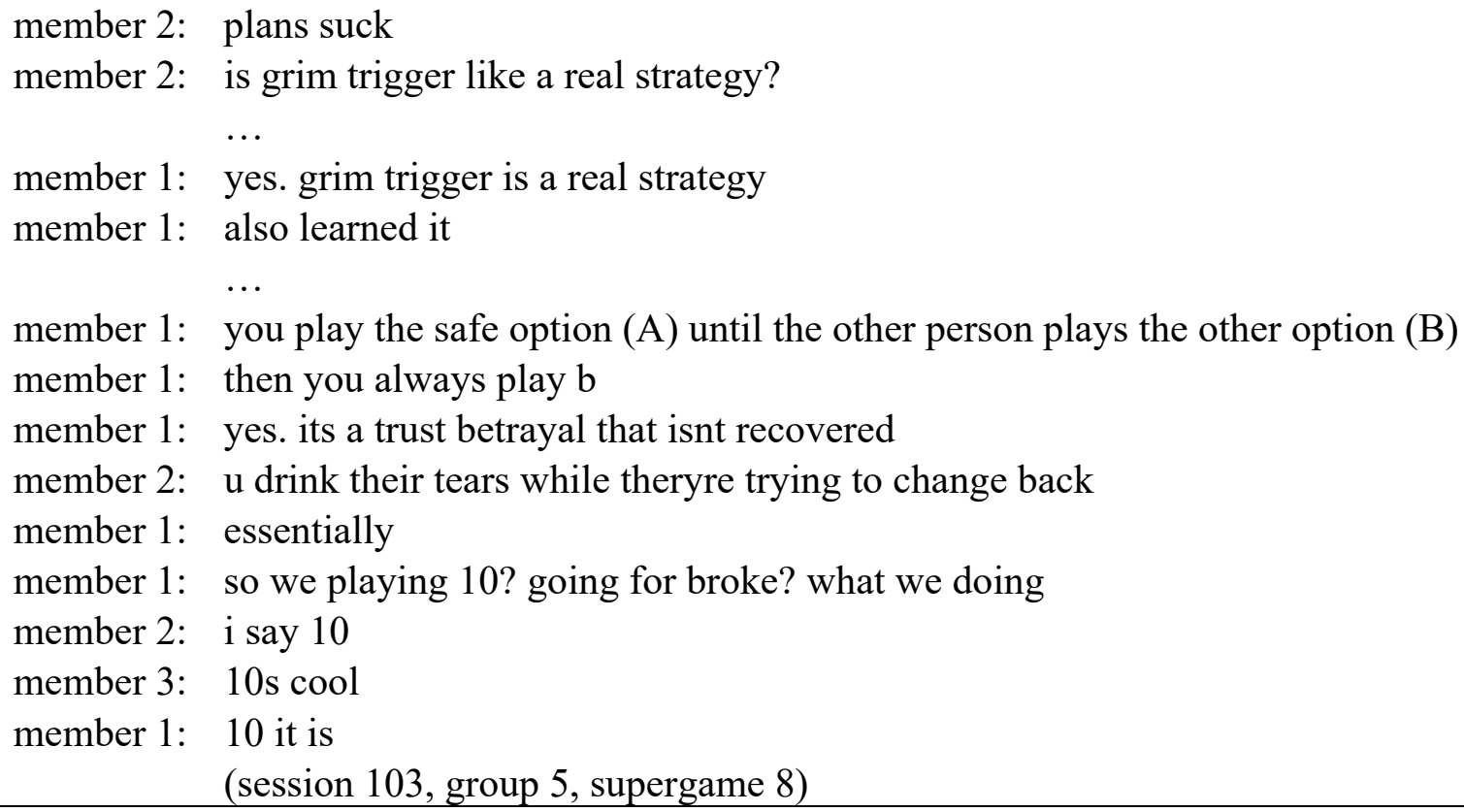

To summarize:

Finding 4: A minority of decision-makers consistently choose Always Defect and are responsible for most of the choices of this poorly-performing strategy. They tend to score worse on the Cognitive Reflection Test, and in the group treatment they refer to game-theoretic reasoning more than others.

\subsection{Group and Individual Cooperation without Noise}

We have focused on the repeated prisoner's dilemma with noise because, as discussed in the introduction, imperfect monitoring is common in many economic interactions. Noise adds complexity to this social dilemma, and we hypothesized that groups may choose different strategies than individuals since they may be more cognitively sophisticated. Removing the noisy implementation of stage game action choices simplifies the strategic environment, and we are aware of no previous studies that use the strategy method to compare group and individual behavior and strategy choices in indefinitely repeated prisoner's dilemmas with perfect monitoring. Therefore, in this subsection we summarize results from additional control treatments that eliminate noise.

These control treatments employ the same stage game payoff parameters as shown in Table 1(a), but with the likelihood of switching a stage game action choice set to 0 . The procedures and instructions were otherwise identical to those described in Section 2, including the same 20 
repeated game strategies (Table 2) available during supergames 5-14. We collected data from 64 subjects, 48 in the group treatment and 16 in the individual treatment, providing two matching groups of eight DMs in each treatment. ${ }^{21}$

Figure 5: No-Noise Stage Game Cooperation Frequency by Supergame

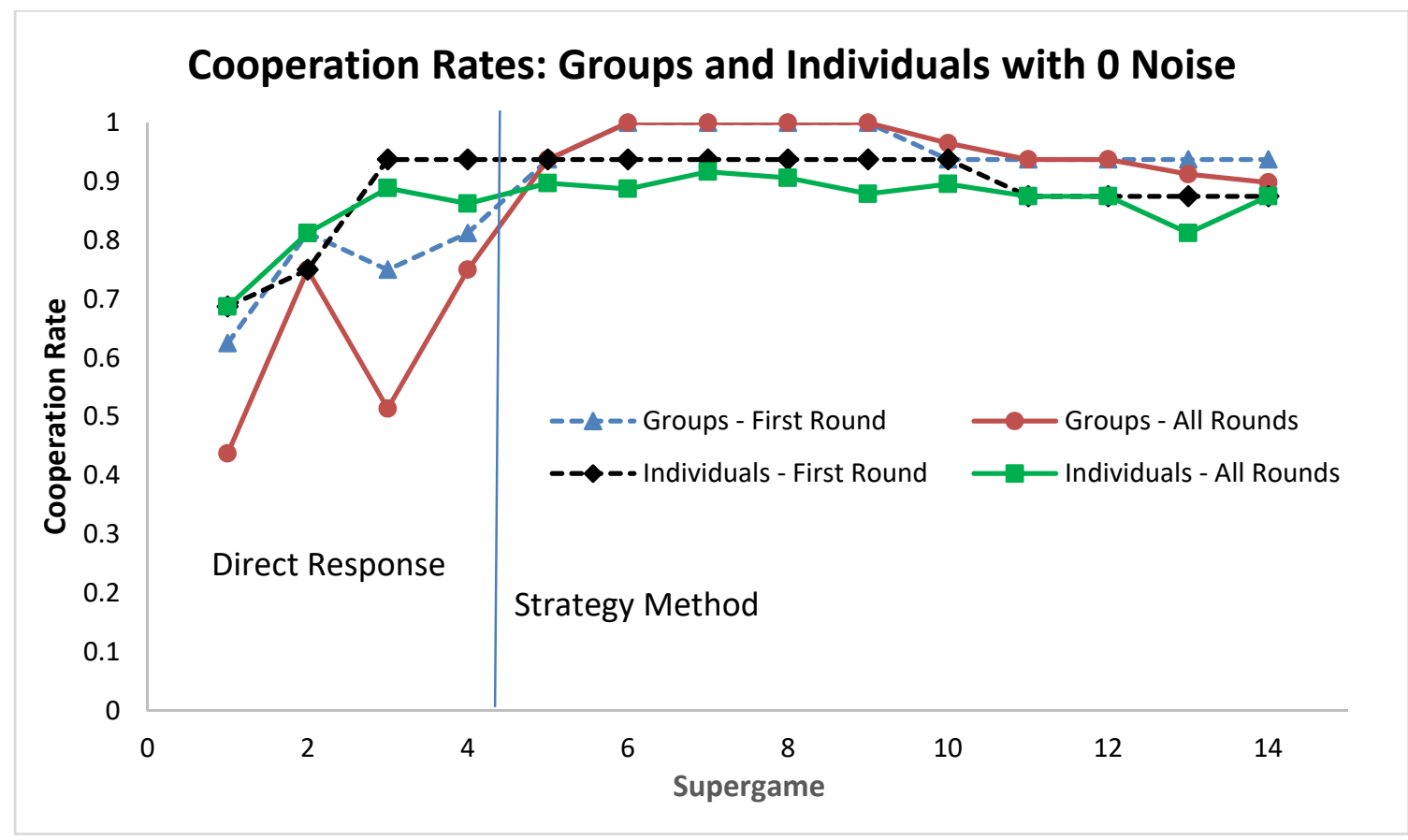

The results of these control treatments are straightforward to summarize. Figure 5 shows that cooperation rates increase across the initial (direct response) supergames in both the individual and group treatments. Individuals tend to cooperate at a higher rate than groups in these initial rounds, and this difference is significant for the four direct response supergames. ${ }^{22}$ Beginning with the first strategy method choice in supergame 5, however, both groups and individuals cooperate at very high rates, always exceeding 80 percent and typically greater than 90 percent cooperation. No statistically significant difference between groups and individuals exists for strategy method

\footnotetext{
${ }^{21}$ FRD also include a control treatment without noise for these same payoff parameters, employing 48 subjects, in their direct response method study of individual decision-makers.

${ }^{22}$ Based on a logit panel regression with random decision-maker effects and standard errors clustered on sessions, $\mathrm{p}$ value $=0.044$ for the first rounds of the direct response supergames and $p$-value $<0.01$ for all rounds of the direct response supergames.
} 
supergames (every comparison p-value exceeds 0.18 ). Groups select variants of TFT in 85 out of 160 strategy choices (53 percent), and individuals select variants of TFT in 97 out of 160 strategy choices (61 percent). Versions of Grim are also similar for groups $(67 / 160=42$ percent) and individuals $(51 / 160=32$ percent). Since DMs selected cooperation-sustaining strategies 93 to 95 percent of the time, cooperative strategies were usually matched together. This allowed players to sustain uniform cooperation in most supergames. Note that even though DMs employ a mixture of TFT and Grim strategies, when matched together in this noiseless environment these strategies are observationally equivalent and so they can only be distinguished using this strategy method elicitation procedure. The strategy method also allows us to observe that subjects rarely change strategy choices, since they repeat the same choice as the previous supergame about 95 percent of the time. ${ }^{23}$

This high level of cooperation is consistent with the notion of a basin of attraction of ALLD introduced by Dal Bó and Fréchette (2011). We chose the Fudenberg et al. (2012) payoff parameters that had the greatest benefit/cost ratio $(b / c=4)$ to generate reasonably high cooperation rates when choices were implemented with noise. For these payoffs in the noise-free environment, a cooperation-sustaining strategy such as Grim is preferred over ALLD if a decision-maker believes the opponent will choose a cooperative strategy with at least 0.048 probability. This basin of attraction for cooperation is even larger than the most favorable game considered by Dal Bó and Fréchette (2011), for which the basin of attraction of Grim over ALLD is a belief that the opponent plays Grim with at least 0.148 probability. In this favorable game their individuals, who played with the direct response method, cooperated over 80 percent of the time in later supergames.

In summary, in the infinitely repeated deterministic PD considered here, individuals and groups behave similarly. Both cooperate at a high rate and seldom change their strategies. Adding noise to the infinitely repeated prisoner's dilemma changes the behavior of both individuals and groups, but the changes for both DMs are such that individuals and groups also behave quite similarly in the presence of noise. With noise Always Defect is the most popular strategy for both individuals and groups. Though groups employ forgiving strategies and TFT strategies more than

\footnotetext{
${ }^{23}$ Groups select ALLD in only one of the 160 strategy choices. One individual, however, chose ALLD in all 10 supergames, and he was the only individual who ever chose ALLD. Consistent with the results in the previous subsection, this individual answered only one of the three CRT questions correctly. Although this one individual regularly chose ALLD while no groups did, the sample size is too small to draw statistically meaningful conclusions about the lower ALLD choice frequency for groups.
} 
individuals, as discussed earlier they also more frequently choose TFT strategies with longer punishment phases. Overall, cooperation rates do not differ across individuals and groups, and both groups and individuals do not experiment much with different strategies. ${ }^{24}$

While this initial exploration of group and individual play of the indefinitely repeated prisoner's dilemma is informative, a more systematic exploration would require a range of games, such as the six payoff and continuation probability conditions explored by Dal Bó and Fréchette (2011). Group decision-making requires many more individual subjects than experiments exclusively focused on individual decision-making, so the data collection requirements are substantial and beyond the scope of the present study. For example, to obtain similar power to Dal Bó and Fréchette (2011) across the same set of six prisoner's dilemma games, comparing individuals to three-person groups would require more than 1000 subjects.

\section{Conclusions}

Many economic relationships have the structure of an infinitely repeated noisy prisoner's dilemma in which the intended actions are implemented with noise. Given the complexity of the social dilemma, learning is likely to be important in affecting decision-makers' choices of repeated game strategies. Decision-makers who seldom experiment with different strategies, however, are unlikely to get useful information that facilitates learning. A good understanding of whether and how players experiment with different repeated game strategies is therefore an important step for understanding how learning affects behavior. To study how experimentation with different strategies affects observed behavior in the repeated noisy prisoner's dilemma, this experiment employs a strategy method design to observe directly how decision-makers change their chosen strategies across supergames. Motivated by the fact that many repeated interactions involve decision-makers who are groups and the empirical findings that overall, groups are cognitively

\footnotetext{
${ }^{24}$ Gong et al. (2009) show that in the finitely repeated deterministic PD, groups cooperate less than individuals. On the other hand, in the finitely repeated stochastic PD described in footnote 8 above, groups cooperate more than individuals. Gong et al. (2009) did not identify the repeated game strategies used by DMs, hence, it is not possible to determine whether groups experiment more than individuals in their study. Besides the difference between finite and infinite repetition, their stage game also differs from our noisy PD. In our noisy PD, a DM's chosen action can be switched randomly to the other action, but the opponent only observes the realized action. In their stochastic PD, players observe the action chosen of the opponent, but the chosen actions of the players only determine each player's payoff stochastically. More systematic work will be required to determine how the structure of uncertainty may affect whether and how group play differs from individuals, and a good place to start will be to study an indefinitely repeated stochastic PD with both the individual and group treatments.
} 
more sophisticated than individuals in one-shot games and perform better than individuals in intellective tasks, we also study how individuals and groups may behave differently in their choices of and experimentation with repeated game strategies.

We find that both individuals and groups frequently choose repeated game strategies that are lenient and/or forgiving, and content analysis of the chats by members of groups reveal that they discuss the fact that "choices can be switched to the other action" $18 \%$ of the time. This category is in fact the most frequently discussed among the 32 different categories and subcategories considered in our content analysis. Our experimental setting differs from Fudenberg et al. (2012) and Aoyagi et al. (2018) who use the direct response method and the Strategy Frequency Estimation Method to infer repeated game strategies chosen by individuals in the noisy prisoner's dilemma. Taken together, however, our work and these earlier studies provide mutuallyreinforcing evidence that individuals recognize the benefits of leniency and forgiveness in a noisy environment and use such strategies often. Our novel empirical evidence regarding group play in the repeated noisy prisoner's dilemma suggests that this importance of lenient and forgiving behavior extends from individuals to groups.

Group decision-making, however, does not change the fact that the counter-intuitive strategy Win-Stay-Lose-Shift is rarely chosen despite its theoretical significance. Group decisionmaking also does not lead subjects to choose the poorly-performing Always Defect strategy less than individuals. Using the Strategy Frequency Estimation Method approach, FRD and Aoyagi et al. (2018) find that Always Defect is the most popular strategy adopted by individuals in the direct response method environment. In our strategy method environment, we find that Always Defect is again the most popular choice for both individuals and groups.

We also find that the 13 decision-makers who choose Always Defect in a majority of the supergames on average scored significantly worse on the Cognitive Reflection Test compared to the others who did not choose this strategy frequently. Examination of the chats by groups who persistently chose Always Defect reveal that members of these groups often recognize that defect is the dominant strategy in the one-shot prisoner's dilemma, which lead them to think that "always B [defect] is the way to go." These groups do not seem to recognize how repetition can fundamentally change the nature of the game, and that history-dependent cooperative strategies can potentially lead to significant gains from cooperation. They seldom talk about "Gains from cooperation" or "Learning from past interactions," but often talk about "It is economic 
theory/game theory." Their low rate of experimentation with alternative strategies, lower scores on the Cognitive Reflection Test, and the relative high incidence of discussing "It is economic theory/game theory" suggest that a little bit of understanding plus a tendency of impulsive thinking can be dangerous. These together prevent them from thinking deeper about the problem, and recognize the possibility that it may be worthwhile for them to experiment the profitability of history-dependent cooperative strategies.

To our knowledge, this study is the first that reports decision-makers' experimentation behavior with different strategies in the repeated noisy prisoner's dilemma. The existing studies on repeated deterministic prisoner's dilemma suggest that experience is important in affecting behavior (Dal Bó and Fréchette, 2018a). The rapid decline in the rate of experimentation with different strategies documented in this repeated noisy prisoner's dilemma, however, suggests that experience may be much less important than heterogeneity in initial choices in determining the later strategy choices. The data suggest that while groups may behave somewhat differently than individuals in the infinitely repeated noisy PD (for example, overall groups choose more forgiving strategies, especially those that are exploitive), groups do not experiment more than individuals in this setting.

This first exploration of strategic experimentation in the repeated noisy prisoner's dilemma is of course is just a single study, and more research is needed to understand how experimentation behavior in repeated noisy games varies with the environment. Our current study provides round by round feedback in the strategy method supergames, and partly to ensure that a session will end in a reasonable time and avoid the possibility of cognitive overload, we only conducted 14 supergames in a session. This number of supergames is greater than in Fudenberg et al. (2012), who conducted an average of 8 to 11 supergames in a session. A natural question for future research is how experimentation behavior may change following experience in a much larger number of supergames. There are many ways to investigate this. One possibility is to conduct sessions where all subjects have participated in the repeated noisy prisoner's dilemma experiment recently. This also helps to observe how the distribution of strategies in the "initial choices" chosen by experienced subjects may differ to the initial choices by inexperienced subjects.

Another direction is to consider the effects of different feedback. In our current study, similar to Fudenberg et al. (2012) and the comparable public monitoring treatment in Aoyagi et al. (2018), as well as Embrey et al. (forthcoming)'s study of the infinitely repeated $4 \times 4$ oligopoly 
games, a decision-maker only observes the actual action implemented by the opponent but does not observe the strategy chosen by the opponent. A decision-maker who does not experiment with other strategies never knows the counterfactual payoffs of alternative strategies. At the other extreme, one can consider an environment in which everyone observes the distribution of strategies chosen by the decision-makers and the average payoff of each strategy (or the average payoff of the decision-makers) in the session after each supergame.

Such information allows decision-makers-in particular those who choose Always Defect - to see how their chosen strategies perform compared to other alternatives. This may prompt more of them to experiment with alternative strategies, and may change the pattern of experimentation over time as well as the final distribution of chosen strategies. Furthermore, our current study finds that decision-makers who choose Always Defect rarely experimented with other strategies and also scored significantly worse in the Cognitive Reflection Test (CRT) that measures both a person's cognitive ability and thinking disposition such as the tendency to seek alternative solutions. Future studies could investigate whether CRT scores are still correlated with the tendency of choosing Always Defect in later supergames in environments with richer feedback. It is worth pointing out that many field settings that resemble the repeated noisy prisoner's dilemma may naturally differ in the feedback that decision-makers can get and in other structural factors. Experimental studies can help generate systematic empirical evidence about how such differences in structural factors can affect the rate and pattern of experimentation and initial heterogeneity in the choices of strategies. Such evidence can inform theory building, and theories that can explain the rate and pattern of experimentation and initial heterogeneity in the choices of strategies across a broad set of environments will be important for understanding field behavior. 


\section{References}

Abbink, K., J. Brandts, B. Herrmann and H. Orzen. (2010). "Intergroup Conflict and Intra-group Punishment in an Experimental Contest Game," American Economic Review, 100, 420-447.

Ahn, T., Isaac, R. and T. Salmon. (2011). "Rent Seeking in Groups," International Journal of Industrial Organization, 29, 116-125.

Aoyagi, M., V. Bhaskar and G. Fréchette. (2018). "The Impact of Monitoring in Infinitely Repeated Games: Perfect, Public, and Private,” American Economic Journal: Microeconomics, forthcoming.

Auerswald, H., C. Schmidt, M. Thum and G. Torsvik. (2018). "Teams in a Public Goods Experiment with Punishment," Journal of Behavioral and Experimental Economics, 72, 28-39.

Axelrod, R. (1984). Evolution of Cooperation. New York: Basic Books.

Axelrod, R. (2012). "Launching 'The Evolution of Cooperation,'” Journal of Theoretical Biology, 299, 2124.

Bornstein, G., D. Budescu, T. Kugler and R. Selten. (2008). "Repeated Price Competition between Individuals and between Teams," Journal of Economic Behavior and Organization, 66, 808-821.

Brandts, J. and G. Charness. (2000), "Hot vs. Cold: Sequential Responses in Simple Experimental Games," Experimental Economics, 2, 227-238.

Brandts, J. and G. Charness. (2011). "The Strategy versus the Direct-Response Method: A First Survey of Experimental Comparisons," Experimental Economics, 14, 375-398.

Camera, G., M. Bigoni and M. Casari. (2012). "Cooperative Strategies in Anonymous Economies: an Experiment," Games and Economic Behavior, 75, 570-586.

Cason, T. and V.-L. Mui (1998), "Social Influence in the Sequential Dictator Game," Journal of Mathematical Psychology, 42, 248-265.

Cason, T. and V.-L. Mui. (2015). "Individual versus Group Play in the Repeated Coordinated Resistance Game," Journal of Experimental Political Science, 2, 94-106.

Charness, G. and M. Sutter. (2012a). “Groups Make Better Self-Interested Decisions," Journal of Economic Perspectives, 26, 157-176.

Charness, G. and M. Sutter. (2012b). "Suggested Further Reading. An Annotated List Accompanying 'Groups make Better Self-interested Decisions'," Online Appendix for "Groups Make Better SelfInterested Decisions," Journal of Economic Perspectives, 26, 157-176. Available at https://assets.aeaweb.org/assets/production/articles-attachments/jep/app/2603_charness_sutter_app.pdf.

Cox, C. and B. Stoddard. (2018). "Strategic Thinking in Public Goods Games with Teams," Journal of Public Economics, 161, 31-43. 
Croson, R. and U. Gneezy. (2009). "Gender Differences in Preferences," Journal of Economic Literature, 47, 448-474.

Dal Bó, P. and G. Fréchette. (2011). "The Evolution of Cooperation in Infinitely Repeated Games: Experimental Evidence," American Economic Review, 101, 411-429.

Dal Bó, P. and G. Fréchette. (2018a). "On the Determinants of Cooperation in Infinitely Repeated Games: A Survey," Journal of Economic Literature, 56, 60-114.

Dal Bó, P. and G. Fréchette. (2018b). "Strategy Choice in the Infinitely Repeated Prisoners Dilemma," Working Paper, Brown and New York Universities.

Dreber, A., D. Fudenberg and D. Rand. (2014). "Who Cooperates in Repeated Games: The Role of Altruism, Inequality Aversion, and Demographics," Journal of Economic Behavior and Organization, 98, 41-55.

Embrey, M., G. Fréchette and S. Yuksel. (2018). “Cooperation in the Finitely Repeated Prisoner's Dilemma," Quarterly Journal of Economics, 133, 509-551.

Embrey, M., F. Mengel and R. Peeters. (2016). "Eliciting strategies in Indefinitely Repeated Games of Strategic Substitutes and Complements," Working Paper, Universities of Sussex and Essex and Maastricht University.

Embrey, M., F. Mengel and R. Peeters. (forthcoming). "Strategy Revision Opportunities and Collusion," Experimental Economics, https://doi.org/10.1007/s10683-018-9580-5.

Engle-Warnick, J. and R. Slonim. (2006) "Learning to Trust in Indefinitely Repeated Games," Games and Economic Behavior, 54, 95-114.

Fischbacher, U. (2007). 'z-Tree: Zurich Toolbox for Readymade Economic Experiments,' Experimental Economics, 10, 171-178.

Fréchette, G. and S. Yuksel. (2017). "Infinitely Repeated Games in the Laboratory: Four Perspectives on Discounting and Random Termination," Experimental Economics, 20, 279-308.

Frederick, S. (2005). "Cognitive Reflection and Decision Making," Journal of Economic Perspectives, 19, $25-42$.

Fudenberg, D. and D. Levine. (2016). "Whither Game Theory? Towards a Theory of Learning in Games," Journal of Economic Perspectives, 30, 151-170.

Fudenberg, D. and E. Maskin. (1990). "Evolution and Cooperation in Noisy Repeated Games," American Economic Review, 80, 274-279.

Fudenberg, D., D. Rand and A. Dreber. (2012). "Slow to Anger and Fast to Forgive: Cooperation in an Uncertain World," American Economic Review, 102, 720-749.

Gneezy, U. and J. Potters. (1997). "An Experiment on Risk Taking and Evaluation Periods," Quarterly Journal of Economics, 112, 631-645. 
Gong, M., J. Baron, and H. Kunreuther. (2009). “Group Cooperation under Uncertainty,’ Journal of Risk and Uncertainty, 39, 251-70.

Greiner, B. (2015). "Subject Pool Recruitment Procedures: Organizing Experiments with ORSEE," Journal of the Economic Science Association, 1, 114-125.

Kagel, J. and P. McGee (2016). 'Team versus Individual Play in Finitely Repeated Prisoner Dilemma Games,' American Economic Journal: Microeconomics, 8, 253-276.

Kamei, K. (forthcoming). "Power of Joint Decision-Making in a Finitely-Repeated Dilemma," Oxford Economic Papers.

Kerr, N. and R. Tindale (2004). "Group Performance and Decision-Making," Annual Review of Psychology, 55, 623-655.

Kroll, S., J. List and C. Mason. (2013). “The Prisoner's Dilemma as Intergroup Game: An Experimental Investigation," in List J. and Price, M. (eds.), Handbook on Experimental Economics and the Environment, Edward Elgar: Cheltenham.

Kugler, T., E. Kausel and M. Kocher. (2012). "Are Groups More Rational Than Individuals? A Review of Interactive Decision Making in Groups," Wiley Interdisciplinary Reviews: Cognitive Science, 3, 471-482.

Laughlin, P., B. Bonner and A. Miner. (2002). "Groups Perform Better than the Best Individuals on Lettersto-Numbers Problems," Organizational Behavior and Human Decision Processes, 88, 605-620.

Laughlin, P. and A. Ellis. (1986). "Demonstrability and Social Combination Processes on Mathematical Intellective Tasks,” Journal of Experimental Social Psychology, 22, 177-189.

Laughlin, P., E. Hatch, J. Silver and L. Boh. (2006). "Groups Perform Better than the Best Individuals on Letters-to-Numbers Problems: Effects of Group Size," Journal of Personality and Social Psychology, 90, 644-651.

Laughlin, P., S. VanderStoep and A. Hollingshead. (1991). "Collective versus Individual Induction: Recognition of Truth, Rejection of Error, and Collective Information Processing," Journal of Personality and Social Psychology, 61, 50-67.

Merlo, A. and A. Schotter (1992). "Theory and Misbehavior of First-Price Auctions: Comment," American Economic Review, 82, 1413-1425.

Müller, W. and F. Tan (2013). "Who Acts More Like a Game Theorist? Group and Individual Play in a Sequential Market Game and the Effect of the Time Horizon," Games and Economic Behavior, 82, 658-674.

Nowak, M. and K. Sigmund. (1993). "A Strategy of Win-Stay, Lose-Shift That Outperforms Tit-for-Tat in the Prisoner's Dilemma Game," Nature, 364, 56-58. 
Romero, J. and Y. Rosokha. (2018). "Constructing Strategies in Indefinitely Repeated Prisoner's Dilemma Game,” European Economic Review, 104, 185-219.

Selten, R., M. Mitzkewitz and G. Uhlich. (1997). "Duopoly Strategies Programmed by Experienced Players," Econometrica, 65, 517-556.

Sheremeta, R. (2016). "Impulsive Behavior in Competition: Testing Theories of Overbidding in RentSeeking Contests,” Working Paper, Case Western Reserve University.

Toplak, M., R. West and K. Stanovich. (2011). "The Cognitive Reflection Test as a Predictor of Performance on Heuristics-and-Biases Tasks," Memory and Cognition, 39: 1275-1289.

Wildschut, T. and C. Insko. (2007). "Explanations of Interindividual-Intergroup Discontinuity: A Review of the Evidence," European Review of Social Psychology, 18, 175-211.

Wildschut, T., B. Pinter, J. Vevea, C. Insko and J. Schopler. (2003). "Beyond the Group Mind: A Quantitative Review of the Interindividual-Intergroup Discontinuity Effect," Psychological Bulletin, 129, 698-722.

Xiao, E. and H. Kunreuther, (2016). "Punishment and Cooperation in Stochastic Social Dilemmas," Journal of Conflict Resolution, 60, 670-693. 


\section{Appendix (Not for Publication): Instructions for the Group treatment}

\section{Part 1 Instructions}

This is an experiment in the economics of multi-person strategic decision making. A research foundation has provided funds for this research. If you follow the instructions and make appropriate decisions, you can earn an appreciable amount of money. At the end of today's session, you will be paid in private and in cash.

It is important that you remain silent and do not look at other people's work. If you have any questions, or need assistance of any kind, please raise your hand but do not say anything, and an experimenter will come to you and will answer your question or provide assistance in private. If you talk, laugh, exclaim out loud, etc., you will be asked to leave and you will not be paid. We expect and appreciate your cooperation.

The experiment is divided into four parts. We are now reading the instructions for Part 1, and instructions for the other parts will be made available later. The 24 participants in today's experiment will be randomly placed into 8 groups of 3 people, and these groups of 3 individuals will remain together throughout the experiment. Each group will make decisions numerous times while interacting with other 3-person groups. You will not know the identity of the participants in your group or other groups in any part of the experiment.

You begin the session with 50 units in your account. Units are then added and/or subtracted

to that amount over the course of the session as described below. At the end of the session, the total number of units in your account will be converted into cash at an exchange rate of 15 units $=\mathrm{US} \$ 1$.

The Session:

The session is divided into a series of interactions between your group and another group in the room.

In each interaction, you will interact for a random number of rounds with another group. In each round you and the group you are interacting with can choose one of two actions. Once the interaction ends, your group gets randomly re-matched with another group in the room for another interaction.

The setup will now be explained in more detail. 


\section{The round}

In each round of the experiment, the same two possible actions are available to both your group and the other group you interact with: A or B.

The earnings of the actions (in units, per person)

$\begin{array}{lll} & \text { You } & \text { Each person in the } \\ \text { Action } & \text { will get } & \text { other group will get }\end{array}$
A: $\quad-2$
$+8$
B:
0
0

If your group's action is A then everyone in your group will get -2 units, and everyone in the other group will get +8 units.

If your group's action is B then everyone in your group will get 0 units, and everyone in the other group will get 0 units.

Calculation of your income in each round:

Your income in each round is the sum of two components:

- the number of units you get from the action your group chose.

- the number of units you get from the action the other group chose.

Your round-total income for each possible choice by you and the other person is thus

\begin{tabular}{|c|c|c|c|}
\hline \multirow[b]{2}{*}{ Your } & & \multicolumn{2}{|c|}{ Other group's choice } \\
\hline & & $\mathrm{A}$ & B \\
\hline Group's & A & +6 & -2 \\
\hline Choice & B & +8 & 0 \\
\hline
\end{tabular}

For example:

If your group chooses A and the other group chooses A, everyone in both groups would get +6 units.

If your group chooses A and the other group chooses B, everyone in your group would get -2 units, and everyone in the other group would get +8 units. 
If your group chooses B and the other group chooses A, everyone in your group would get +8 units, and everyone in the other group would get -2 units.

If your group chooses B and the other group chooses B, everyone in both groups would get 0 .

To make your group's choice, you will first indicate your proposed A or B choice on your computer screen, illustrated on the next page. Once everyone in your group has submitted their proposals, everyone in your group will have the opportunity to type chat messages on your computer for 45 seconds to discuss your group decision. Although we will record these messages that you send, only you and the other two people in your group will see them. Note, in sending messages back and forth we request that you follow two simple rules: (1) Be civil to each other and use no profanity and (2) Do not identify yourself. The chat time will be reduced to 30 seconds each round beginning with the $3^{\text {rd }}$ interaction.

After this chat time ends you will then vote for which choice (A or B) you want your group to make for this round. Your group's choice will be determined by majority vote; that is, whichever choice (A or B) receives the most votes will be your choice for the round. The computer program will calculate your income for each round based on your group's choice and the choice of the other group. The choices and your income will be shown on a results screen, illustrated below. The total number of units you have at the end of the session will determine how much money you earn, at an exchange rate of 15 units $=\$ 1$.

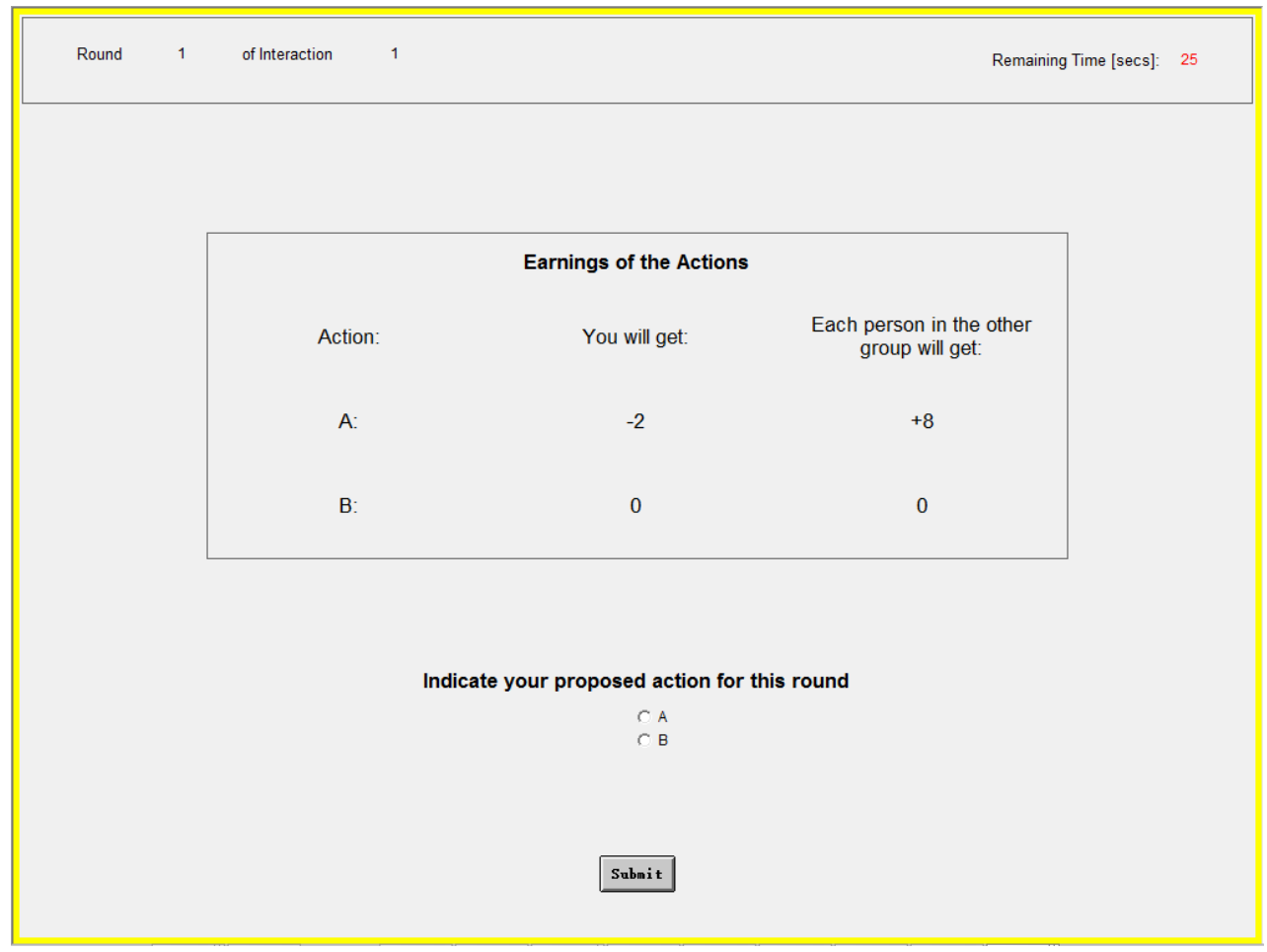



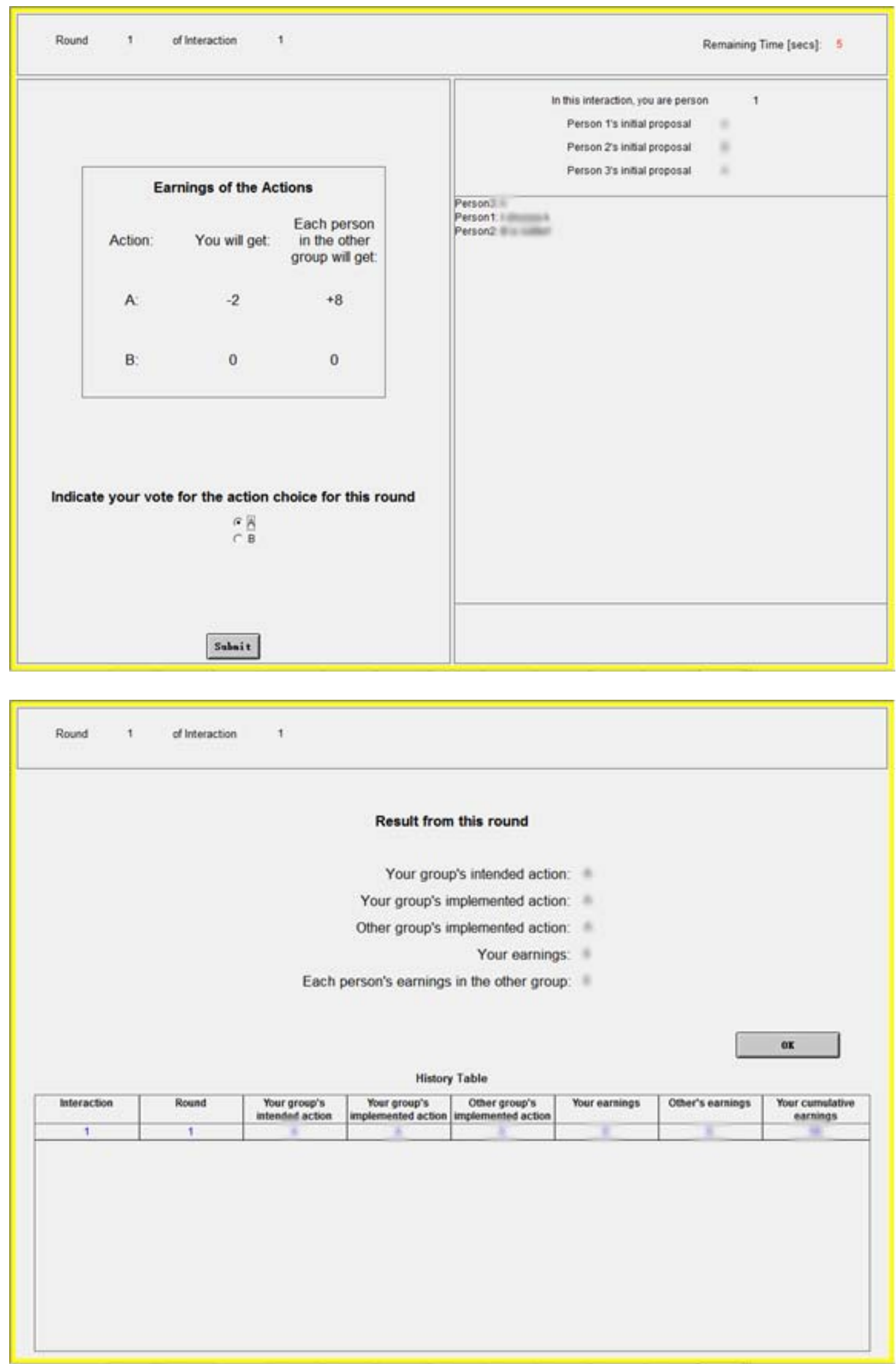


\section{A chance that your group's choice is changed}

There is a $7 / 8$ probability that the action your group chooses actually occurs. But with probability $1 / 8$, your group's action is changed to the opposite of what you picked. That is:

- When your group chooses $\mathrm{A}$, there is a $7 / 8$ chance that the computer will actually implement A as your group's action, and 1/8 chance that instead the computer will implement B as your group's action. The same is true for the other group.

- When your group chooses $\mathrm{B}$, there is a 7/8 chance that the computer will actually implement B as your group's action, and 1/8 chance that instead the computer will implement A as your group's action. The same is true for the other group.

You can think of this probability as the outcome of a roll from an 8-sided die, with sides labelled 1 through 8 . Separately for every group and for every single choice made by that group, the die is rolled and if an 8 appears then the choice is switched to the other action. Otherwise, for the 7 other possible die roll outcomes the choice is implemented as intended. These possible switches are independent for all groups and choices, so you should think of this as a separate die roll for every group in every round.

Both groups are informed of the actions that are actually implemented. Neither group is informed of the intended choice made by the other group. Thus with 1/8 probability, an error in execution occurs, and you never know whether the other group's action was what they chose, or an error.

For example, if your group chooses A and the other group chooses B then:

- With probability $(7 / 8) *(7 / 8)=0.766$, no changes occur. You will both be told that your group's action is A and the other group's action is B. Everyone in your group will get -2 units, and everyone in the other group will get +8 units.

- With probability $(7 / 8)^{*}(1 / 8)=0.109$, the other group's action is changed from their choice. You will both be told that your group's action is A and the other group's action is A. Everyone in both groups will all get +6 units.

- With probability $(1 / 8)^{*}(7 / 8)=0.109$, your group's action is changed from your choice. You will both be told that your group's action is B and the other group's action is B. Everyone in both groups will all get +0 units.

- With probability $(1 / 8)^{*}(1 / 8)=0.016$, both your group's action and the other group's action are changed from your choices. You will both be told that your group's action is B and the 
other group's action is A. Everyone in your group will get +6 units and everyone in the other group will get -2 units.

\section{Random number of rounds in each interaction}

After each round, there is a $7 / 8$ probability of another round, and $1 / 8$ probability that the interaction will end. Successive rounds will occur with probability $7 / 8$ each time, until the interaction ends (with probability 1/8 after each round). You can think of this as a hard spin of a lottery wheel with 8 equally-sized slices, as illustrated below. Only if this spin comes up 8 does the interaction end. To make the experiment run faster, earlier we used a computerized random number generator to simulate repeated spins of this 8-space wheel, and recorded the outcomes to determine the actual random lengths of each interaction. These round lengths are written in the sealed envelope I am holding up now, and this will be opened for inspection at the end of the experiment today.

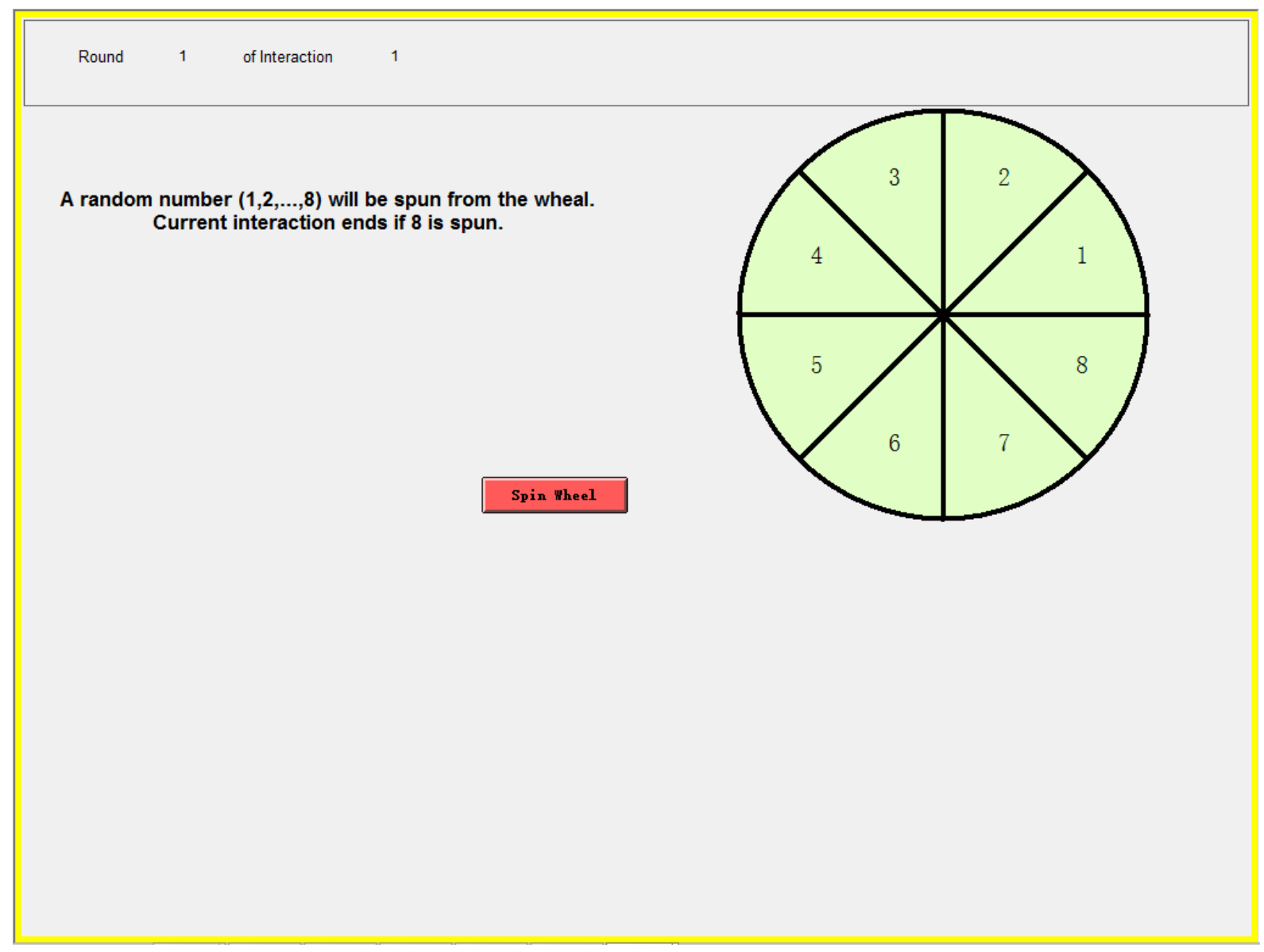


Once each interaction ends, your group will be randomly re-matched with a different group in the room for another interaction. Each interaction has the same setup. You will have a number of such interactions with different groups. Remember that the people in your own 3-person group (and each of the 7 other 3-person groups) remain together in this same group throughout the experiment today.

Your group will not be matched twice with the same group during this part of the session. You will be matched with a new and different group in every single interaction.

\section{Summary}

To summarize, the 24 participants in today's experiment will be randomly placed into 8 groups of 3 people, and these groups of 3 individuals will remain together throughout the experiment.

Every interaction your group has with another group in the experiment includes a random number of rounds. After every round, there is a 7/8 probability of another round. There will be a number of such interactions, and your behavior has no effect on the number of rounds or the number of interactions.

There is a $1 / 8$ probability that the action your group chooses (through majority voting) will not happen and the opposite action occurs instead, and the same is true for the group you interact with. You will be told which actions actually occur, but you will not know what action the other group actually intended.

At the beginning of the session, you have 50 units in your account. At the end of the session, you will receive $\$ 1$ for every 15 units in your account.

Your group will not be matched twice with the same group during this part of the session. Your group will be matched with a new and different group in every single interaction. 


\section{Part 2 Instructions}

Now that you have become familiar with the decision tasks of the experiment, for the next 3 interactions we will have each group choose a plan that avoids the need to make a decision roundby-round. Each of these next 3 interactions will still have the same rules as the first 4 interactions in Part 1 that involved round-by-round decisions. Your group will again choose actions A or B each round, and with probability $1 / 8$ your group's action is changed to the opposite of what you picked. Each interaction will again terminate with probability $1 / 8$ each round. Once each interaction ends, your group will be randomly re-matched with a different group for another interaction. Your group will not be matched twice with the same group during this part of the session, or with any group that you were matched with during Part 1. Your group will be matched with a new, different group in every single interaction.

\section{The Possible Plans}

For each interaction you can choose from one of 20 possible plans, first indicating your proposed plan on a screen as illustrated below.

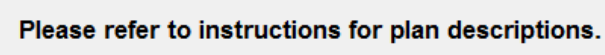

Indicate your proposed plan for this interaction.

$$
\begin{aligned}
& \text { C Plan } 1 \\
& \text { C Plan } 2 \\
& \text { c Plan } 3 \\
& \text { C Plan } 4 \\
& \text { C Plan } 5 \\
& \text { c Plan } 6 \\
& \text { C Plan } 7 \\
& \text { C Plan } 8 \\
& \text { c Plan } 9 \\
& \text { C Plan } 10 \\
& \text { C Plan } 11 \\
& \text { C Plan } 12 \\
& \text { C Plan } 13 \\
& \text { C Plan } 14 \\
& \text { C Plan } 15 \\
& \text { C Plan } 16 \\
& \text { C Plan } 17 \\
& \text { C Plan } 18 \\
& \text { C Plan } 19 \\
& \text { C Plan } 20
\end{aligned}
$$


Once everyone in your group has submitted their proposals, everyone in your group will have the opportunity to type chat messages on your computer for 360 seconds (6 minutes) to discuss your group's plan. Again, we will record these messages that you send, but only you and the other two people in your group will see them. As before, when sending messages back and forth we request that you follow two simple rules: (1) Be civil to each other and use no profanity and (2) Do not identify yourself. The chat time will be reduced to 5 and then 4 minutes in subsequent interactions.

After this chat time ends you will then vote for which plan you want your group to make for this interaction, as shown below. Your group's choice will be determined by majority vote; that is, whichever plan receives the most votes will be your choice for the round. In the event that three different plans each receive one vote, one of the three voted plans will be chosen randomly for the interaction.

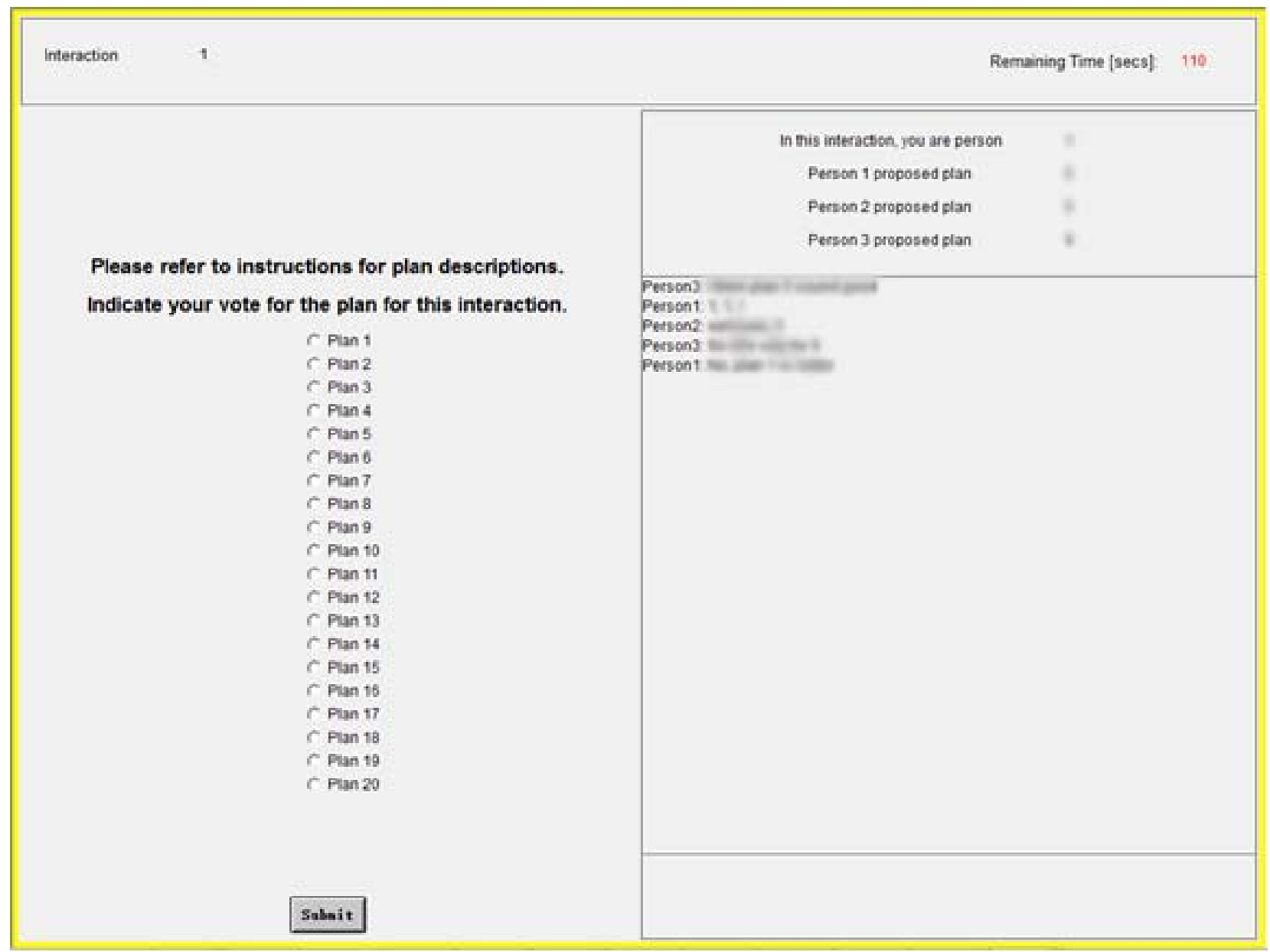


After you have voted for your plan for the interaction, you will receive a confirmation screen that restates your voted plan and gives you an opportunity to revise it if you have made a mistake.

Once your group has chosen a plan for the interaction, this plan cannot be changed in later rounds within that interaction. Some plans specify different actions based on the outcomes of previous rounds. We will first describe plans that start round 1 by choosing action A, and then we will describe plans that start by choosing action B. Note that whenever the description prescribes a choice other than A, it implements a choice of B (since that is the only other choice available in a round).

Plan 1. Always choose A in all rounds.

Plan 2. Start by choosing A, then always choose A unless the other group's action is B in the previous round.

Plan 3. Start by choosing A, then always choose A unless the other group's action is B in the two previous rounds.

Plan 4. Start by choosing A, then always choose A unless the other group's action is B in the three previous rounds.

Plan 5. Start by choosing A, then always choose A unless the other group's action is B in either of the two previous rounds. If your group's choice is B because the other group's action was B previously, then always choose two consecutive rounds of B; but switch back to A if, and only if, the other group's actions are two consecutive rounds of A.

Plan 6. Start by choosing A, then always choose A unless the other group's action is B in two out of the previous three rounds. If your group's choice is B because the other group's actions were two consecutive $\mathrm{B}$ actions, then always choose two consecutive rounds of B before switching back to choose A.

Plan 7. Start by choosing A, then continue choosing A until either your group's action or the other group's action is B in the previous round. If this occurs, then choose B twice before switching back to choose A.

Plan 8. Start by choosing A, and choose A whenever both group's actions match (A-A or B$\mathrm{B}$ ) in the previous round; otherwise choose $\mathrm{B}$.

Plan 9. Start by choosing A, and choose A whenever both group's actions match (A-A or BB) for two consecutive previous rounds; otherwise choose B.

The following 4 plans also start with A, but they indicate that once a switch to B is chosen then B will be chosen thereafter for the remainder of the interaction. 
Plan 10. Start by choosing A, and continue to choose A until either group's action is B in the previous round. If either group's previous action is B, then choose B for every remaining round of the interaction.

Plan 11. Start by choosing A, and continue to choose A until either group's action is B for two consecutive previous rounds. If this occurs, then choose $\mathrm{B}$ for every remaining round of the interaction.

Plan 12. Start by choosing A, and continue to choose A until either group's action is B for three consecutive previous rounds. If this occurs, then choose B for every remaining round of the interaction.

Plan 13. Start by choosing A, then choose B for every remaining round of the interaction.

The remaining 7 possible plans start with action $B$ in round 1 .

Plan 14. Always choose $B$ in all rounds.

Plan 15. Start by choosing B, then always choose A unless the other group's action is B in the previous round.

Plan 16. Start by choosing B, then always choose A unless the other group's action is B in the two previous rounds.

Plan 17. Start by choosing B, then always choose A unless the other group's action is B in the three previous rounds.

Plan 18. Start by choosing B, then switch to choose A until either group's action is B for two consecutive previous rounds. If this occurs, then choose B for every remaining round of the interaction.

Plan 19. Start by choosing B, then switch to choose A until either group's action is B for three consecutive previous rounds. If this occurs, then choose B for every remaining round of the interaction

Plan 20. Start by choosing B, then switch to A, then switch to B, etc., alternating between A and $\mathrm{B}$ for every round of the interaction, regardless of what the other group's actions are in previous rounds.

After you and the other group choose your plans, you will be shown the outcome of each round of the interaction, including your group's intended action (based on your group's plan), your group's implemented action, the other group's implemented action, and the result of the wheel spin that determines whether the current interaction is continued or terminated. An example screen is shown on the next page. 


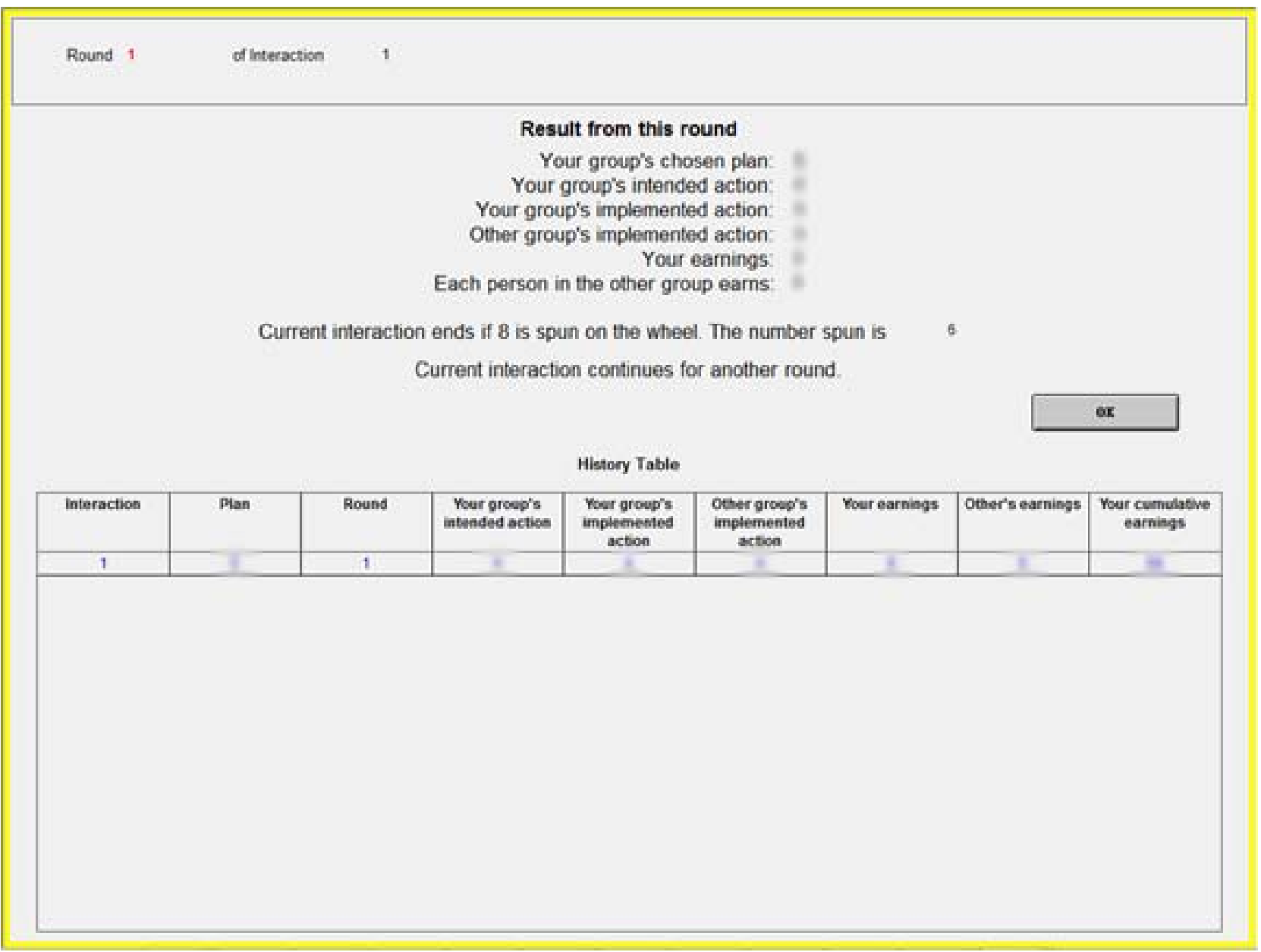




\section{Part 3 Instructions}

You have now completed 7 interactions, one with each of the 7 other groups participating in today's experiment. For Part 3 of the experiment, you will again be matched once and only once with each of the 7 other groups for one interaction each. The order in which you are matched with each other group for these interactions is random and it differs from the order during Parts 1 and 2. The interactions will take place exactly like Part 2, with each group choosing a plan to implement for the entire interaction. The only difference is that we will shorten the chat time at the start of each interaction to 3 minutes.

The number of rounds for each interaction is random again, with each interaction ending with probability $1 / 8$ at the end of each round. These random draws are independent of the draws from Parts 1 -2, and were determined with additional wheel spins. The actual random lengths of each interaction are also written in the sealed envelope that will be opened for inspection at the end of the experiment today.

After this part there will be no more interactions with others in the experiment. Part 4 (to be described later) includes a questionnaire and some short, simple decision tasks that each of you will undertake separately, without any interaction with others. 